ALEA, Lat. Am. J. Probab. Math. Stat. 18, 1689-1718 (2021)

DOI: $10.30757 /$ ALEA.v18-62

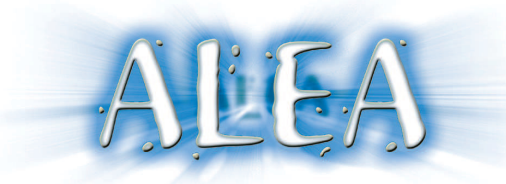

\title{
Extremes of the 2d scale-inhomogeneous discrete Gaussian free field: Extremal process in the weakly correlated regime
}

\author{
Maximilian Fels, Lisa Hartung \\ M. Fels, Institut für Angewandte Mathematik, Rheinische Friedrich-Wilhelms-Universität \\ Endenicher Allee 60, 53115 Bonn, Germany \\ E-mail address: fels@iam.uni-bonn.de \\ $U R L$ : wt.iam.uni-bonn.de/maximilian-fels \\ L. Hartung, Institut für Mathematik, Johannes Gutenberg-Universität Mainz \\ Staudingerweg 9, 55099 Mainz, Germany \\ E-mail address: lhartung@uni-mainz.de \\ URL: https://sites.google.com/view/lisahartung/home
}

\begin{abstract}
We prove convergence of the full extremal process of the scale-inhomogeneous discrete Gaussian free field in dimension two in the weak correlation regime. The scale-inhomogeneous discrete Gaussian free field is obtained from the $2 \mathrm{~d}$ discrete Gaussian free field by modifying the variance through a function $\mathcal{I}:[0,1] \rightarrow[0,1]$. The full extremal process converges to a cluster Cox process. The random intensity of the Cox process depends on $\mathcal{I}^{\prime}(0)$ through a random measure $Y$ and on $\mathcal{I}^{\prime}(1)$ through a constant $\beta$. We show that, in law, the random measure, $Y$, is equal to the Liouville Quantum Gravity measure at sub-critical temperature $\alpha=2 \sigma(0)$. The cluster process, which only depends on $\mathcal{I}^{\prime}(1)$, can be described as atoms of a standard $2 \mathrm{~d}$ discrete Gaussian free field conditioned to be unusually high.
\end{abstract}

\section{Introduction}

Log-correlated processes have received a lot of attention in recent years, see e.g. Aïdékon et al. (2013); Arguin et al. (2013); Ding et al. (2017); Biskup and Louidor (2018); Bovier and Hartung (2014); Mallein (2015); Fyodorov (2004); Maillard and Zeitouni (2016); Arguin et al. (2017, 2019). Prominent examples are branching Brownian motion (BBM), the two-dimensional discrete Gaussian free field (DGFF), cover times of Brownian motion on the torus, characteristic polynomials of

Received by the editors June 30th, 2020; accepted May 21th, 2021.

2010 Mathematics Subject Classification. 60G15,60G60,60G70.

Key words and phrases. Gaussian free field, inhomogeneous environment, extreme values, extremal processes, branching Brownian motion, branching random walk.

The research of M.F. is partially funded by the Deutsche Forschungsgemeinschaft (DFG, German Research Foundation) under Germany's Excellence Strategy - GZ 2047/1, Projekt-ID 390685813 and GZ 2151 - Project-ID 390873048, through the Collaborative Research Center 1060 The Mathematics of Emergent Effects. The research of L. H. is supported in part by the Deutsche Forschungsgemeinschaft (DFG, German Research Foundation) through Project-ID 233630050 -TRR 146, Project-ID 443891315 within SPP 2265 and and Project-ID 446173099. 
random unitary matrices or local maxima of the randomized Riemann zeta function on the critical line. One of the key features in these models is that their correlations are such that they start to become relevant for the extreme values of the processes. In particular, one is interested in the structure of the extremal processes that arises when the size of the index set tends to infinity. In the case of the $2 \mathrm{~d}$ DGFF, one considers the field indexed by the vertices of a lattice box of side length $N$, where $N$ is taken to infinity. In this paper, we study the extremal process of the scaleinhomogeneous $2 \mathrm{~d}$ DGFF in the weakly correlated regime. The model first appeared as a tool to prove Poisson-Dirichlet statistics of the extreme values of the 2d DGFF (Arguin and Zindy, 2015). In the context of the $2 \mathrm{~d}$ DGFF, it is the natural analogue model of variable-speed BBM or the timeinhomogeneous branching random walk (BRW). We start with a precise definition of the model we consider in the following.

Definition 1.1 (2d discrete Gaussian free field (DGFF)). Let $N \in \mathbb{N}$ and $V_{N}=[0, N)^{2} \cap \mathbb{Z}^{2}$. Then, the centred Gaussian field $\left\{\phi_{v}^{N}\right\}_{v \in V_{N}}$ with correlations given by the Green kernel

$$
\mathbb{E}\left[\phi_{v}^{N} \phi_{w}^{N}\right]=G_{V_{N}}(v, w):=\frac{\pi}{2} \mathbb{E}_{v}\left[\sum_{k=0}^{\tau_{\partial V_{N}}-1} \mathbb{1}_{S_{k}=w}\right] \text {, for } v, w \in V_{N}
$$

is called DGFF on $V_{N}$. Here, $\mathbb{E}_{v}$ is the expectation with respect to the simple random walk (SRW) $\left\{S_{k}\right\}_{k \geq 0}$ on $\mathbb{Z}^{2}$ started in $v$ and $\tau_{\partial V_{N}}$ denotes the stopping time of the SRW hitting the boundary $\partial V_{N}$. Note our particular choice of scaling for the Green function.

Next, we define the 2d scale-inhomogeneous DGFF.

Definition 1.2 (2d scale-inhomogeneous DGFF). Let $\left\{\phi_{v}^{N}\right\}_{v \in V_{N}}$ be a DGFF on $V_{N}$. For $v=$ $\left(v_{1}, v_{2}\right) \in V_{N}$ and $\lambda \in(0,1)$, set

$$
[v]_{\lambda} \equiv[v]_{\lambda}^{N}:=\left(\left[v_{1}-\frac{1}{2} N^{1-\lambda}, v_{1}+\frac{1}{2} N^{1-\lambda}\right] \times\left[v_{2}-\frac{1}{2} N^{1-\lambda}, v_{2}+\frac{1}{2} N^{1-\lambda}\right]\right) \cap V_{N} .
$$

We set $[v]_{0}^{N}:=V_{N}$ and $[v]_{1}^{N}:=\{v\}$. We denote by $[v]_{\lambda}^{o}$ the interior of $[v]_{\lambda}$. Let $\mathcal{F}_{\partial[v]_{\lambda} \cup[v]_{\lambda}^{c}:=}$ $\sigma\left(\left\{\phi_{v}^{N}, v \notin[v]_{\lambda}^{o}\right\}\right)$ be the $\sigma$-algebra generated by the random variables outside $[v]_{\lambda}^{o}$. For $v \in V_{N}$, let

$$
\phi_{v}^{N}(\lambda)=\mathbb{E}\left[\phi_{v}^{N} \mid \mathcal{F}_{\partial[v]_{\lambda} \cup[v]_{\lambda}^{c}}\right], \quad \lambda \in[0,1] .
$$

We denote by $\nabla \phi_{v}^{N}(\lambda)$ the increment, $\lim _{h \rightarrow 0} \phi_{v}^{N}(\lambda+h)-\phi_{v}^{N}(\lambda)$, of the DGFF at vertex $v$ and scale $\lambda$, which simply is a discrete increment. Moreover, let $s \mapsto \sigma(s)$ be a non-negative function such that $\mathcal{I}_{\sigma^{2}}(\lambda):=\int_{0}^{\lambda} \sigma^{2}(x) \mathrm{d} x$ is a function on $[0,1]$ with $\mathcal{I}_{\sigma^{2}}(0)=1$ and $\mathcal{I}_{\sigma^{2}}(1)=1$. The $2 \mathrm{~d}$ scale-inhomogeneous DGFF on $V_{N}$ is a centred Gaussian field, $\psi^{N}:=\left\{\psi_{v}^{N}\right\}_{v \in V_{N}}$, defined as

$$
\psi_{v}^{N}:=\int_{0}^{1} \sigma(s) \nabla \phi_{v}^{N}(s) \mathrm{d} s .
$$

For $\delta>0$, let $V_{N}^{\delta}=[\delta N,(1-\delta) N)^{2} \cap \mathbb{Z}^{2}$. Fels and Hartung (2019, Lemma 3.3 (ii)) shows that it is a centred Gaussian field with covariance given by

$$
\mathbb{E}\left[\psi_{v}^{N} \psi_{w}^{N}\right]=\log N \mathcal{I}_{\sigma^{2}}\left(\frac{\log N-\log _{+}\|v-w\|_{2}}{\log N}\right)+O(1), \quad \text { for } v, w \in V_{N}^{\delta},
$$

with $\log _{+}=\max \{0, \log (x)\}$.

Note that when choosing $\mathcal{I}_{\sigma^{2}}(x) \equiv x$, the field, $\left\{\psi_{v}^{N}\right\}_{v \in V_{N}}$, is the usual DGFF as in Definition 1.1. In this paper we consider the weak correlation regime, in which the correlations of $\left\{\psi_{v}^{N}\right\}_{v \in V_{N}}$ are smaller than in the usual DGFF. 
Assumption 1.3 (Assumption of weak correlations). In the rest of the paper, $\left\{\psi_{v}^{N}\right\}_{v \in V_{N}}$ is always a 2d scale-inhomogeneous DGFF on $V_{N}$. We assume that $\mathcal{I}_{\sigma^{2}}(x)<x$, for $x \in(0,1)$, and that $\mathcal{I}_{\sigma^{2}}(1)=1$. Moreover, we assume that $s \mapsto \sigma^{2}(s)$ is differentiable at 0 and 1 , and satisfies $\sigma(0)<1$ and $\sigma(1)>1$.

Under Assumption 1.3 we proved in Fels (2019); Fels and Hartung (2019), building on work by Arguin and Ouimet Arguin and Ouimet (2016), the sub-leading order correction, tightness and convergence of the appropriately centred maximum. More explicitely, there exists a constant, $\beta=$ $\beta(\sigma(1))$, which depends only on the final variance $\sigma^{2}(1)$, and a random variable, $Y=Y(\sigma(0))$, depending only on the initial variance $\sigma^{2}(0)$, such that, for any $z \in \mathbb{R}$,

$$
\lim _{N \rightarrow \infty} \mathbb{P}\left(\max _{v \in V_{N}} \psi_{v}^{N} \leq m_{N}-z\right)=\mathbb{E}\left[\exp \left[-\beta Y e^{-2 z}\right]\right],
$$

where $m_{N}:=2 \log N-\frac{\log \log N}{4}$. In particular, the limiting law solely depends on $\sigma(0)$ and $\sigma(1)$ and is therefore universal in the considered regime. Note that $m_{N}$ is also the order of the maximum of $N^{2}$ iid centred Gaussians with variance $\log N$, in which case the law of the centered maximum is simply Gumbel (see Bovier, 2017, Theorem 1.2). In particular, it differs from the centring of the maximum value in case of the usual $2 \mathrm{~d}$ DGFF, in which one observes an additional logarithmic correction such that the correct centring in this case is $2 \log N-\frac{3 \log \log N}{4}$ (see Bramson and Zeitouni, 2012). Moreover, we proved in Fels and Hartung (2019, Theorem 2.2) that under Assumption 1.3, points whose height is close to the maximum are either $O(N)$ apart or within distance $O(1)$. In particular, there is a constant $c>0$, such that

$$
\lim _{r \rightarrow \infty} \lim _{N \rightarrow \infty} \mathbb{P}\left(\exists u, v \in V_{N} \text { with } r \leq\|u-v\|_{2} \leq \frac{N}{r} \text { and } \psi_{u}^{N}, \psi_{v}^{N} \geq m_{N}-c \log \log r\right)=0 .
$$

To state our results, we introduce some additional notation. Let $A \subset[0,1]^{2}$ and $B \subset \mathbb{R}$ be two Borel sets. For $v \in \mathbb{Z}^{2}$ and $r>0$, let its $r$-neighbourhood be $\Lambda_{r}(v)=\left\{w \in \mathbb{Z}^{2}:\|v-w\|_{2} \leq r\right\}$. Then, define

$$
\eta_{N, r}(A \times B):=\sum_{v \in V_{N}} \mathbb{1}_{\psi_{v}^{N}=\max _{u \in \Lambda_{r}(v)} \psi_{u}^{N}} \mathbb{1}_{x / N \in A} \mathbb{1}_{\psi_{v}^{N}-m_{N} \in B} .
$$

$\eta_{N, r}$ is a point measure encoding both position and relative height of extreme local maxima in $r$ neighbourhoods. To study distributional limits of these point measures, we equip the space of point measures on $[0,1]^{2} \times \mathbb{R}$ with the vague topology. Our first result is convergence of $\eta_{N, r}$ to a Poisson point process $(\mathrm{PPP})$ with a random intensity measure. We set $\overline{\mathbb{R}}=\mathbb{R} \cup\{\infty\}$.

Theorem 1.4. Let $\left\{\psi_{v}^{N}\right\}_{v \in V_{N}}$ be a scale-inhomogeneous DGFF satisfying Assumption 1.3. Then, there is a random measure $Y(\mathrm{~d} x)$ on $[0,1]^{2}$ which depends only on the initial variance $\sigma(0)$ and satisfies almost surely $Y\left([0,1]^{2}\right)<\infty$ and $Y(A)>0$, for any open and non-empty $A \subset[0,1]^{2}$. Moreover, there is a constant $\beta=\beta(\sigma(1))>0$, depending only on the final variance $\sigma(1)$, such that, for any sequence $r_{N}$ with $r_{N} \rightarrow \infty$ and $r_{N} / N \rightarrow 0$, as $N \rightarrow \infty$,

$$
\eta_{N, r_{N}} \stackrel{N \rightarrow \infty}{\rightarrow} \operatorname{PPP}\left(Y(\mathrm{~d} x) \otimes \beta e^{-2 h} \mathrm{~d} h\right)
$$

where convergence is in law with respect to the vague convergence of Radon measures on $[0,1]^{2} \times \overline{\mathbb{R}}$.

Moreover, the random measure, $Y(\mathrm{~d} x)$, can be characterized more explicitly. For each $\alpha \in[0, \infty)$, define the sequence of random measures,

$$
\varphi_{N}^{\alpha}(\mathrm{d} x):=\mathbb{1}_{[0,1]^{2}}(x) \exp \left[\alpha \phi_{\lfloor x N\rfloor}^{N}-\frac{\alpha^{2}}{2} \mathbb{E}\left[\left(\phi_{\lfloor x N\rfloor}^{N}\right)^{2}\right]\right] \mathrm{d} x
$$

By Kahane (1985) and Shamov (2016), there exists a random, almost surely finite Borel measure, $\varphi_{\infty}^{\alpha}$, called Gaussian multiplicative chaos associated with the continuum Gaussian free field, also 
known as Liouville Quantum Gravity measure. It is concentrated on $[0,1]^{2}$ and such that, for any measurable set $A \subset[0,1]^{2}$,

$$
\lim _{N \rightarrow \infty} \varphi_{N}^{\alpha}(A)=\varphi_{\infty}^{\alpha}(A) \quad \text { a.s. }
$$

It is known (Rhodes and Vargas, 2014, Theorem 5.5) that, for each $\alpha \in(0,2)$, we have almost surely, $\varphi_{\infty}^{\alpha}\left([0,1]^{2}\right)>0$.

Theorem 1.5. Let $s \mapsto \mathcal{I}_{\sigma^{2}}(s)$ and the random measure, $Y(\sigma(0))(\mathrm{d} x)$, be as in Theorem 1.4. Then, there exists a constant, $c \in(0, \infty)$, such that, in distribution,

$$
Y(\sigma(0))(\mathrm{d} x)=c \varphi_{\infty}^{2 \sigma(0)}(\mathrm{d} x)
$$

In particular, $Y$ has the law of the Liouville Quantum Gravity measure on $[0,1]^{2}$ corresponding to (sub-critical) parameter $\alpha=2 \sigma(0)$.

We remark that Biskup and Louidor (2019) provide an alternative description of the Liouville Quantum gravity measure at sub-critical temperature, $\alpha \in(0,2)$, via the random measure encoding the location of the intermediate level sets of the usual DGFF, which are composed of the vertices which lie a fraction $\alpha / 2$ above the global maximum. In particular, they identify a number of axiomatic properties of this measure which uniquely characterize its law (Biskup and Louidor, 2019, Theorem 2.3). In the critical case, when $\alpha=2, \varphi_{\infty}^{2}$ was identified by Biskup and Louidor (2018) with the so-called derivative martingale, the random intensity measure governing the extremal process of the 2d DGFF.

As the field at nearby vertices is strongly correlated, around each local maximum there will naturally be plenty of particles being close to it. Together with location and height of $r$-local maxima, we encode them in the point process

$$
\mu_{N, r}:=\sum_{v \in V_{N}} \mathbb{1}_{\psi_{v}^{N}=\max _{u \in \Lambda_{r}(v)} \psi_{u}^{N}} \delta_{x / N} \otimes \delta_{\psi_{v}^{N}-m_{N}} \otimes \delta_{\left\{\psi_{v}^{N}-\psi_{v+w}^{N}: w \in \mathbb{Z}^{2}\right\}} .
$$

These are Radon measures on $[0,1]^{2} \times \mathbb{R} \times \mathbb{R}^{\mathbb{Z}^{2}}$. We consider this space equipped with the topology of vague convergence. The following theorem shows convergence of $\mu_{N, r}$, the full extremal process.

Theorem 1.6. There is a probability measure $\theta$ on $[0, \infty)^{\mathbb{Z}^{2}}$ such that for each $r_{N}$ with $r_{N} \rightarrow \infty$ and $r_{N} / N \rightarrow 0$, as $N \rightarrow \infty$,

$$
\mu_{N, r_{N}} \rightarrow P P P\left(Y(\mathrm{~d} x) \otimes \beta e^{-2 h} \mathrm{~d} h \otimes \Xi(\mathrm{d} \nu)\right) .
$$

The convergence is in law with respect to the vague convergence of Radon measures on $[0,1]^{2} \times \overline{\mathbb{R}} \times$ $\overline{\mathbb{R}}^{\mathbb{Z}^{2}}$. Moreover, $\Xi$ is given by the weak limit,

$$
\begin{aligned}
\Xi(A)= & \lim _{r \rightarrow \infty} \mathbb{P}\left(\left(\phi_{v}^{\mathbb{Z}^{2} \backslash\{0\}}+2 \sigma(1) \mathfrak{a}(v)\right)_{v \in \mathbb{Z}^{2}} \in A \mid \phi_{w}^{\mathbb{Z}^{2} \backslash\{0\}}+2 \sigma(1) \mathfrak{a}(w) \geq 0, \forall\|w\|_{2} \leq r\right), \\
& \forall A \in \mathcal{B}\left([0, \infty)^{\mathbb{Z}^{2}}\right)
\end{aligned}
$$

with

$$
\mathfrak{a}(w)=\lim _{N \rightarrow \infty} G_{V_{2 N}}[(N, N),(N, N)]-G_{V_{2 N}}[(N, N),(N, N)+w]
$$

being the potential kernel and where the field, $\left\{\phi_{x}^{\mathbb{Z}^{2} \backslash\{0\}}: x \in \mathbb{Z}^{2}\right\}$, is the DGFF on $\mathbb{Z}^{2} \backslash\{0\}$. In addition, $\Xi_{0}=0$ and $\left|\left\{w \in \mathbb{Z}^{2}: \nu_{w} \leq c\right\}\right|<\infty, \Xi-$ a.s. for each $c>0$. 
Note that the field, $\left\{\phi_{x}^{\mathbb{Z}^{2} \backslash\{0\}}: x \in \mathbb{Z}^{2}\right\}$, is also referred to as the pinned field, that is the DGFF on $\mathbb{Z}^{2}$ pinned to zero at the origin. In particular, it is a centred Gaussian field with correlations given by

$$
\mathbb{E}\left[\phi_{x}^{\mathbb{Z}^{2} \backslash\{0\}} \phi_{y}^{\mathbb{Z}^{2} \backslash\{0\}}\right]=\mathfrak{a}(x)+\mathfrak{a}(y)-\mathfrak{a}(x-y),
$$

with $x \mapsto \mathfrak{a}(x)$ being the potential kernel as in (1.16). The latter admits the asymptotic form, $\mathfrak{a}(x)=\log \|x\|_{2}+O(1)$, as $\|x\|_{2} \rightarrow \infty$ (see Lawler and Limic, 2010, Theorem 4.4.4). Regarding the measure, $\mu_{N, r_{N}}$, as defined in (1.13), one would naturally want to write the cluster law, i.e. its third coordinate, as a probability measure on the configurations of the field, $\left\{\psi_{u}^{N}-\psi_{u+w}^{N}\right.$ : $\left.w \in \mathbb{Z}^{2},\|w-v\|_{2} \leq r_{N}\right\}$, when conditioning $u$ to be an extreme local maximum. Considering the fact that the cluster law only sees the field in a "local" neighbourhood of an extreme local maximum and due to the regularity of the variance at these fine scales (see Assumption 1.3), one can replace the field $\left\{\psi_{v}^{N}: v \in V_{N}\right\}$ by the scaled 2d DGFF, $\left\{\sigma(1) \phi_{v}^{N}: v \in V_{N}\right\}$. Conditioning on both position and height of the corresponding extreme local maximum, and shifting its position to the origin, we can replace the $2 \mathrm{~d}$ DGFF by the pinned field with a determinstic shift, $2 \sigma(1) \mathfrak{a}(w)$, for $w \in \mathbb{Z}^{2}$. The shift reflects the fact that the height of the extreme local maximum at the origin is roughly $m_{N}$ and the variance of the field being $\sigma(1)$. The condition to have an extreme local maximum at the origin translates into the condition in (1.15). As a consequence of Theorem 1.6, we obtain convergence of the extremal process

$$
\eta_{N}:=\sum_{v \in V_{N}} \delta_{v / N} \otimes \delta_{\psi_{v}^{N}-m_{N}}
$$

to a cluster Cox process.

Corollary 1.7. Let $\left\{\left(x_{i}, h_{i}\right): i \in \mathbb{N}\right\}$ enumerate the points in a sample of $P P P\left(Y(\mathrm{~d} x) \otimes \beta e^{-2 h} \mathrm{~d} h\right)$. Let $\left\{\theta_{w}^{(i)}: w \in \mathbb{Z}^{2}\right\}, i \in \mathbb{N}$, be independent samples from the measure $\Xi$, independent of $\left\{\left(x_{i}, h_{i}\right)\right.$ : $i \in \mathbb{N}\}$. Then, as $N \rightarrow \infty$,

$$
\eta_{N} \rightarrow \sum_{i \in \mathbb{N}} \sum_{w \in \mathbb{Z}^{2}} \delta_{\left(x_{i}, h_{i}-\theta_{w}^{(i)}\right)} .
$$

The convergence is in law with respect to the vague convergence of Radon measures on $[0,1]^{2} \times \overline{\mathbb{R}}$. Moreover, the measure on the right-hand side of (1.19) is locally finite on $[0,1]^{2} \times \mathbb{R}$ a.s.

1.1. Related work. Choosing $\sigma(x) \equiv 1$, for $x \in[0,1]$, in (1.4) gives the $2 \mathrm{~d}$ DGFF. Its maximum value was investigated by Bolthausen et al. (2001); Daviaud (2006); Bolthausen et al. (2011); Bramson and Zeitouni (2012); Ding (2013); Ding and Zeitouni (2014); Bramson et al. (2016), which culminated in the proof of convergence of the maximum Bramson et al. (2016). Biskup and Louidor proved convergence of the extremal point process encoding local maxima and the field centred at those, to a cluster Cox process (Biskup and Louidor, 2016, 2018). The random intensity measure is identified with the so-called Liouville quantum gravity measure (Biskup and Louidor, 2019). The cluster law of the $2 \mathrm{~d}$ DGFF admits a closely related formulation to the one we obtain in Theorem 1.6, namely

$$
\begin{aligned}
\Xi_{D G F F}(A)= & \lim _{r \rightarrow \infty} \mathbb{P}\left(\left(\phi_{v}^{\mathbb{Z}^{2} \backslash\{0\}}+2 \mathfrak{a}(v)\right)_{v \in \mathbb{Z}^{2}} \in A \mid \phi_{w}^{\mathbb{Z}^{2} \backslash\{0\}}+2 \mathfrak{a}(w) \geq 0, \forall\|w\|_{1} \leq r\right), \\
& \forall A \in \mathcal{B}\left([0, \infty)^{\mathbb{Z}^{2}}\right) .
\end{aligned}
$$

The slight, however important difference, is that the factor $\sigma(1)$ in $(1.15)$ is equal to one. This causes the conditioning in (1.20) to be asymptotically singular. There is another possible regime in the scale-inhomogeneous DGFF, i.e. when $\mathcal{I}_{\sigma^{2}}(x)>x$, for some $x \in(0,1)$. When $x \mapsto \mathcal{I}_{\sigma^{2}}(x)$ is piecewise linear, the leading and sub-leading order of the maximum, as well as exponential tails of the centred maximum, in particular tightness, are known (Arguin and Ouimet, 2016; Fels, 2019). 
Variable-speed branching Brownian motion (BBM), which first appeared in Derrida and Spohn (1988), is the natural analogue in the context of BBM of the scale-inhomogeneous DGFF. It is a centred Gaussian process indexed by the leaves of the super-critical Galton-Watson tree, and covariance given by $t A(d(v, w) / t)$, where $d(v, w)$ is the time of the most recent common ancestor of two leaves $v$ and $w$. The function, $x \mapsto A(x)$, in variable-speed BBM takes the role of the map, $x \mapsto \mathcal{I}_{\sigma^{2}}(x)$, in the case of the scale-inhomogeneous DGFF. $A(x) \equiv 1$ corresponds to standard BBM. Its extremal process was investigated in Aïdékon et al. (2013); Arguin et al. (2013); Bovier and Hartung (2017); Bramson (1978); Lalley and Sellke (1987); Arguin et al. (2011, 2012); Cortines et al. (2019). In Aïdékon et al. (2013); Arguin et al. (2013), the cluster process was shown to be $\mathrm{BBM}$ conditioned on the maximum being larger than $\sqrt{2} t$, or alternatively given as the limiting distribution of the neighbours of a local maximum. The extremal process of variable-speed BBM was investigated in Bovier and Hartung (2014, 2015); Maillard and Zeitouni (2016); Fang and Zeitouni (2012); Bovier and Hartung (2020). In the regime of weak correlations, i.e. when $A(x)<x$, for $x \in(0,1), A^{\prime}(0)<1$ and $A^{\prime}(1)>1$, Bovier and Hartung $(2014,2015)$ proved convergence of the extremal process to a cluster Cox process. The cluster law can be described by the law of BBM in time $t$, conditioned on the maximum being larger than $\sqrt{2} A^{\prime}(1) t$, which is a perfect match to the one in the weakly correlated regime of the scale-inhomogeneous DGFF in (1.15). In the regime when $A$ is strictly concave, Bovier and Kurkova (2004) showed that the first order of the maximum depends only on the concave hull of $A$. Moreover, Maillard and Zeitouni (2016) proved that the 2nd order correction is proportional to $t^{1 / 3}$.

Note that there are other models such as the BRW (Mallein, 2018) or first passage percolation (Kistler et al., 2020) where it was proven that the extremal process converges to a (cluster) Cox process.

1.2. Outline of Proof. We start to explain the proof of Theorem 1.4. First, we deduce tightness of $\eta_{N, r}$ from (1.6), (1.7) and a uniform exponential upper bound on extreme level sets, which is proven in Proposition 2.1. Then, we characterize possible limit laws as a Cox processes using a superposition principle as in Biskup and Louidor (2016). Finally, we need to show uniqueness of the random intensity measure. This follows from the convergence in distribution of multiple local maxima over disjoint subsets (see Theorem 2.5, proven in Section 4). The proof of Theorem 2.5 relies on ideas from the proof of convergence of the global maximum Fels and Hartung (2019). We give a detailed outline of the proof of Theorem 2.5 at the beginning of Section 4. In particular, Theorem 1.5 can be easily deduced from it.

Next, we explain the proof of Theorem 1.6. By (1.7), we know that extreme local maxima have to be separated at distance $O(N)$ and, due to correlations, are surrounded by $O(1)$ neighbourhoods of high points. We need to show that the $O(1)$ neighbourhoods of extreme local maxima converge to independent samples of a cluster law. Using (1.7) we know that also the $O(1)$ neigbourhoods must be at macroscopic distance, i.e. at distance of $O(N)$. To obtain independence of the clusters, we decompose the field into a sum of independent "local fields" that are zero outside the $O(1)$ neighbourhoods and a "binding field", which captures the contributions from outside the neighbourhoods. The requirement of being a cluster around a local maximum then translates into the local field being smaller than the value at its centre. We then show convergence of the laws of the local fields conditioned on a local maximum at their centre. In particular, we deduce that the clusters are i.i.d. samples of a common cluster law. Together with convergence of the extremal process of local maxima, Theorem 1.4, this yields Theorem 1.6.

Structure of the paper: In Section 2, we prove Theorem 1.4. The necessary ingredient, convergence of multiple local maxima over disjoint subsets, i.e. Theorem 2.5, and the identification of the random measure with the sub-critical Liouville Quantum Gravity measure, i.e. Theorem 1.5, are proved in Section 4. The proof of Theorem 1.6 is provided in Section 3. The appendix recalls Gaussian comparison tools. 


\section{Proof of Theorem 1.4}

It turns out that we are able to follow and use large parts of the proof for the DGFF by Biskup and Louidor (2016). As depicted in Biskup and Louidor (2016); Bovier (2017), the fact that the limiting point process takes the particular form of a generalized Poisson point process, is a consequence of a superposition property, which is due to its Gaussian nature along with certain properties of the field such as the separation of local maxima (Fels and Hartung, 2019) and tightness of extreme level sets. The main ingredient we need, in order to apply the machinery from Biskup and Louidor (2016) to obtain the distributional invariance and thus Poisson limit laws, is tightness of the point processes, which is a consequence of the following proposition and previous results in Fels and Hartung (2019). For $y \in \mathbb{R}$, we denote by

$$
\Gamma_{N}(y)=\left\{v \in V_{N}: \psi_{v}^{N} \geq m_{N}-y\right\}
$$

the level set above $m_{N}-y$.

Proposition 2.1. There exists a constant $C>0$, such that, for all $z>1$ and all $\kappa$,

$$
\sup _{N \geq 1} \mathbb{P}\left(\left|\Gamma_{N}(y)\right|>e^{\kappa z}\right) \leq C e^{2 y-\kappa z}
$$

Proof: By a first order Chebychev inequality, using that the variance of $\psi_{v}^{N}$, for $v \in V_{N}$, is bounded uniformly by $\log N+o(1)$ (see (1.5)) and a standard Gaussian tail bound,

$$
\mathbb{P}\left(\left|\Gamma_{N}(y)\right|>e^{\kappa z}\right) \leq \tilde{C} \frac{\sqrt{\log N}}{m_{N}} N^{2} \exp \left[-\frac{\left(m_{N}-y\right)^{2}}{2 \log N}-\kappa z\right] \leq C \exp [2 y-\kappa z],
$$

which shows (2.2).

Proposition 2.1 together with Fels and Hartung (2019, Theorem 2.1) implies tightness of $\left\{\eta_{N, r_{N}}\right\}_{N \in \mathbb{N}}$, as the right-hand side of (2.2) tends to zero as $\kappa \rightarrow \infty$.

2.1. Distributional Invariance. Let $\left(W_{t}\right)_{t \geq 0}$ be an independent standard Brownian motion started in 0 . Given a measurable function $f:[0,1] \times \mathbb{R} \rightarrow[0, \infty)$, let

$$
f_{t}(x, h)=-\log \mathbb{E}^{0}\left[e^{-f\left(x, h+W_{t}-\frac{1}{2} t\right)}\right], \quad t \geq 0,
$$

where $\mathbb{E}^{0}$ is the expectation with respect to the Brownian motion $\left(W_{t}\right)_{t \geq 0}$.

Theorem 2.2. (cp. Biskup and Louidor, 2016, Theorem 3.1) Let $\eta$ be any sub-sequential distributional limit of the processes $\left\{\eta_{N, r_{N}}\right\}_{N \geq 1}$, for some $r_{N} \rightarrow \infty$ with $r_{N} / N \rightarrow 0$. Then, for any continuous function $f:[0,1]^{2} \times \mathbb{R} \rightarrow[0, \infty)$ with compact support and all $t \geq 0$,

$$
\mathbb{E}\left[e^{-<\eta, f>}\right]=\mathbb{E}\left[e^{-<\eta, f_{t}>}\right] .
$$

Proof: The proof of Theorem 2.2 is a rerun of the one in the case of the 2d DGFF (Biskup and Louidor, 2016, Theorem 3.1). We therefore omit details here. It essentially uses convergence of the maximum obtained in Fels and Hartung (2019) together with exponential bounds on level sets, see Proposition 2.1.

Remark 2.3. As we think that the interpretation of the statement by Biskup and Louidor (2016) is enlightening, we reproduce it here. Picking a sample, $\eta$, of the limit process, we know by tightness that $\eta(C)<\infty$ almost surely for any compact $C$. This allows us to write

$$
\eta=\sum_{i \in \mathbb{N}} \delta_{\left(x_{i}, h_{i}\right)},
$$


where $\left\{\left(x_{i}, h_{i}\right) \in[0,1] \times \mathbb{R} \cup\{-\infty\}: i \in \mathbb{N}\right\}$ enumerate the points. Let $\left\{W_{t}^{(i)}: i \in \mathbb{N}\right\}$ be a collection of independent standard Brownian motions, independent of $\eta$, and set

$$
\eta_{t}:=\sum_{i \in \mathbb{N}} \delta_{\left(x_{i}, h_{i}+W_{t}^{(i)}-\frac{1}{2} t\right)}, \quad t \geq 0 .
$$

Using Fubini and dominated convergence, we have for all non-negative functions $f$,

$$
\mathbb{E}\left[e^{-<\eta, f_{t}>}\right]=\mathbb{E}\left[e^{-<\eta_{t}, f>}\right] .
$$

Theorem 2.2 then implies,

$$
\eta_{t} \stackrel{d}{=} \eta, \quad t \geq 0
$$

We borrow from Biskup and Louidor (2016) a short heuristic argument why Theorem 2.2 should hold. Let $\psi$ be a scale-inhomogeneous DGFF on $V_{N}$ satisfying Assumption 1.3 and let $\psi^{\prime}, \psi^{\prime \prime}$ be two independent copies of it. Fix some $t>0$. Then,

$$
\psi \stackrel{d}{=} \sqrt{1-\frac{t}{\log N}} \psi^{\prime}+\sqrt{\frac{t}{\log N}} \psi^{\prime \prime}=\psi^{\prime}-\frac{t}{2 \log N} \psi^{\prime}+\sqrt{\frac{t}{\log N}} \psi^{\prime \prime}+o(1),
$$

where we have used a Taylor expansion of the first square root, which has an error term $O\left(t^{2} / \log ^{2} N\right)$. Using the fact, that the first order of the maximum of the scale-inhomogeneous DGFF is $\log N$, we obtain an error $o(1)$. If we take $v \in V_{N}$ away from the boundary, where $\psi_{v} \geq m_{N}-y$ or $\psi_{v}^{\prime} \geq m_{N}-y$ and consider the $r$-neighbourhood $\Lambda_{r}(v)$, we first note that, for $w \in \Lambda_{r}(v), \psi_{w}^{\prime \prime}-\psi_{v}^{\prime \prime}=O(1)$, and so by the prefactor, we may write,

$$
\psi_{w} \stackrel{d}{=} \psi_{w}^{\prime}-\frac{t}{2 \log N} \psi_{w}^{\prime}+\sqrt{\frac{t}{\log N}} \psi_{v}^{\prime \prime}+o(1), \quad w \in \Lambda_{r}(v)
$$

Similarly, we know that $\psi_{w}-m_{N}=O(1)$ and $\psi_{w}^{\prime}-m_{N}=O(1)$, for $w \in \Lambda_{r}(v)$, and thus, we may replace $\frac{t}{2 \log N} \psi_{w}^{\prime}$ by $\frac{t}{2 \log N}\left(m_{N}+O(1)\right)=t+o(1)$, to obtain

$$
\psi_{w} \stackrel{d}{=} \psi_{w}^{\prime}-t+\sqrt{\frac{t}{\log N}} \psi_{v}^{\prime \prime}+o(1), \quad w \in \Lambda_{r}(v) .
$$

Finally, we see that $\sqrt{\frac{t}{\log N}} \psi^{\prime \prime}$ is asymptotically distributed as $W_{t}$, where $\left(W_{t}\right)_{t \geq 0}$ is a Brownian motion. Further, we know from Fels and Hartung (2019, Theorem 2.2), that local extremes are at distance of order $N$ and so the field $\psi^{\prime \prime}$ in two such neighbourhoods has correlation of order $O(1)$. The normalizing factor $\sqrt{\frac{t}{\log N}}$ then implies that two such neighbourhoods are asymptotically independent. Thus, for $N$ large, we have a one-to-one correspondence between local maxima of $\psi$ and local maxima of $\psi^{\prime}$ by a shift in their height through independent Brownian motions with drift -1 .

2.2. Poisson limit law. Just as in Biskup and Louidor (2016), distributional invariance, Theorem 2.2, allows to extract a Poisson limit law for every such subsequence, i.e. for any sub-sequential limit of the extremal process. In our setting, we can directly apply Biskup and Louidor (2016, Theorem 3.2).

Theorem 2.4. Biskup and Louidor (2016, Theorem 3.2) Suppose that $\eta$ is a sub-sequential limit of the process $\eta_{N, r_{N}}$, that is a point process on $[0,1]^{2} \times \mathbb{R}$ such that, for some $t>0$, and all continuous functions $f:[0,1]^{2} \times \mathbb{R} \rightarrow[0, \infty)$ with compact support, it holds, as in Theorem 2.2,

$$
\mathbb{E}\left[e^{-<\eta, f>}\right]=\mathbb{E}\left[e^{-<\eta, f_{t}>}\right] .
$$


Moreover, assume that almost surely $\eta\left([0,1]^{2} \times[0, \infty)\right)<\infty$ and $\eta\left([0,1]^{2} \times \mathbb{R}\right)>0$. Then, there is a random Borel measure $Y$ on $[0,1]^{2}$, satisfying $Y\left([0,1]^{2}\right) \in(0, \infty)$ almost surely, such that

$$
\eta \stackrel{d}{=} P P P\left(Y(\mathrm{~d} x) \otimes \beta e^{-2 h} \mathrm{~d} h\right) .
$$

2.3. Uniqueness. In this section, we show uniqueness of the extremal process of local extremes, i.e. of the limit $\lim _{N \rightarrow \infty} \eta_{N, r_{N}}$. In light of Theorem 2.4, we do this by showing uniqueness of the random measure $Y(\mathrm{~d} x)$. The proof is a generalization of the proof of uniqueness of the random variable $Y$ in Fels and Hartung (2019, Theorem 2.1). We show that the joint law of local maxima converges in law and that this law can be written as a Laplace transform of the random measure $Y(\mathrm{~d} x)$, which then implies uniqueness of $Y(\mathrm{~d} x)$. For a set $A \subset[0,1]$, we write $\psi_{N, A}^{*}=\max \left\{\psi_{v}^{N}: v \in V_{N}, v / N \in A\right\}$.

Theorem 2.5. Let $\left(A_{1}, \ldots, A_{p}\right)$ be a collection of disjoint non-empty open subsets of $[0,1]^{2}$. Then the law of $\left(\max \left\{\psi_{v}^{N}: v \in V_{N}, v / N \in A_{l}\right\}-m_{N}\right)_{l=1}^{p}$ converges weakly as $N \rightarrow \infty$. More precisely, there are random variables $Y_{A_{1}}, \ldots, Y_{A_{p}}$ depending only on the initial variance $\sigma(0)$, satisfying $Y_{A_{i}}>$ 0 almost surely, for $1 \leq i \leq p$, and there is a constant $\beta>0$, depending only on the final variance $\sigma(1)$, such that

$$
\lim _{N \rightarrow \infty} \mathbb{P}\left(\psi_{N, A_{l}}^{*}-m_{N} \leq x_{l}: l=1, \ldots, p\right)=\mathbb{E}\left[\exp \left(-\beta \sum_{l=1}^{p} e^{-2 x_{l}} Y_{A_{l}}\right)\right] .
$$

The constant $\beta$ in Theorem 2.5 is identical to the one appearing in (1.6). Next, we prove Theorem 1.4. The proof of Theorem 2.5 is given in Section 4 .

Proof of Theorem 1.4 using Theorem 2.5: Let $r_{N} \rightarrow \infty$ with $r_{N} / N \rightarrow 0$ be now a fixed sequence. Denote by $\eta$ a corresponding sub-sequential limit of the extremal process $\left\{\eta_{N, r_{N}}\right\}_{N \geq 1}$. By Theorem 2.4, there is a corresponding random measure $\tilde{Y}(\mathrm{~d} x)$ such that $\eta \stackrel{d}{=} P P P\left(\tilde{Y}(\mathrm{~d} x) \otimes \beta e^{-2 h} \mathrm{~d} h\right)$. Note that, as a trivial consequence of Theorem 2.5, for any open and non-empty $A \subset[0,1]^{2}$, $\psi_{N, A}^{*}-m_{N}$ is a tight sequence. Fix an arbitrary collection, $\left(A_{1}, \ldots, A_{p}\right)$, of disjoint, open and non-empty subsets of $[0,1]^{2}$, with $\tilde{Y}\left(\partial A_{l}\right)=0$, for any $l \in\{1, \ldots, p\}$. By Theorem 2.5 , there is a dense subset $R \subset \mathbb{R}$ such that, for any $x_{1}, \ldots, x_{p} \in R$,

$$
\mathbb{E}\left[\exp \left(-\beta \sum_{l=1}^{p} e^{-2 x_{l}} \tilde{Y}\left(A_{l}\right)\right)\right]=\lim _{N \rightarrow \infty} \mathbb{P}\left(\psi_{N, A_{l}}^{*}-m_{N} \leq x_{l}: l=1, \ldots, p\right) .
$$

Again by Theorem 2.5, the right-hand side of (2.16) is the same for all subsequences. Using continuity in $x$ of the left hand side, we can deduce from convergence on the dense subset $R$, convergence on $\mathbb{R}$. Along with a standard approximation argument of continuous functions on $[0,1]^{2}$ via nonnegative simple functions, this implies uniqueness of the Laplace transform of the random measure $\tilde{Y}(\mathrm{~d} x)$ on the disjoint collection $\left(A_{1}, \ldots, A_{p}\right)$, regardless of the subsequence considered. As $p \in \mathbb{N}$ and $A_{1}, \ldots, A_{p}$ are arbitrary, it follows that $\tilde{Y}(\mathrm{~d} x)$ is the same for all sub-sequences. Therefore, we obtain a random Borel measure $Y(\mathrm{~d} x)$ whose masses of any countable collection of open sets $A_{1}, \ldots, A_{p}$ are given by $Y_{A_{1}}, \ldots, Y_{A_{p}}$ from Theorem 2.5, depending only on $\sigma(0)$. We conclude, that the law of the measure $Y(\mathrm{~d} x)$ also depends only on initial variance, $\sigma(0)$. Further, note that by Proposition 2.1,

$$
\mathbb{P}\left(\eta\left([0,1]^{2} \times[-y, \infty]\right)>e^{k y}\right) \leq C e^{-y(\kappa-2)} .
$$

In combination with Theorem 2.4, (2.17) implies that the total mass of $Y$ is almost surely finite. In addition, if we choose as test function, $f \in C_{c}\left([0,1]^{2} \times\{\mathbb{R} \cup\{\infty\}\}\right)$ with $f \geq 0$ and $\operatorname{supp}(f) \subset$ 
$[0,1]^{2} \times[t, \infty]$, then the identity

$$
\left\{\max _{v \in V_{N}} \psi_{v}^{N}<m_{N}+t\right\}=\left\{\eta_{N, r_{N}}\left([0,1]^{2} \times[t, \infty)\right)=0\right\}
$$

together with (2.17) and $\kappa=0$ implies that $\eta$ is concentrated on $[0,1]^{2} \times \mathbb{R}$. Moreover, Theorem 2.5 implies that, for any non-empty and open $A \subset[0,1]^{2}$, we have almost surely $Y(A)>0$.

\section{Proof of Theorem 1.6}

In the following, we assume that $V_{N}$ is centred at the origin. Let $\mu$ be a Radon measure on $[0,1]^{2} \times \mathbb{R} \times \mathbb{R}^{\mathbb{Z}^{2}}$ and $f:[0,1]^{2} \times \mathbb{R} \times \overline{\mathbb{R}}^{\mathbb{Z}^{2}} \rightarrow[0, \infty)$ be a measurable function with compact support. We write

$$
\langle\mu, f\rangle:=\int \mu(\mathrm{d} x \mathrm{~d} h \mathrm{~d} \theta) f(x, h, \theta)
$$

Further, let

$$
\Theta_{N, r}:=\left\{v \in V_{N}: \psi_{v}^{N}=\max _{u \in \Lambda_{r}(v)} \psi_{u}^{N}\right\}
$$

be the set of $r$-local maxima.

Lemma 3.1. For any $r_{N} \rightarrow \infty$ with $r_{N} / N \rightarrow 0$ and any continuous function $f:[0,1]^{2} \times \mathbb{R} \times \mathbb{R}^{\mathbb{Z}^{2}}$ with compact support,

$$
\lim _{r \rightarrow \infty} \limsup _{N \rightarrow \infty} \max _{M: r \leq M \leq N / r}\left|\mathbb{E}\left[e^{-\left\langle\mu_{N, r_{N}}, f\right\rangle}\right]-\mathbb{E}\left[e^{-\left\langle\mu_{N, M}, f\right\rangle}\right]\right|=0 .
$$

Proof: Let $\lambda>0$ be such that $f(x, h, \theta)=0$, for $h \geq \lambda$. If $\left\langle\mu_{N, r_{N}}, f\right\rangle \neq\left\langle\mu_{N, M}, f\right\rangle$, for some $M$ with $r \leq M \leq N / r$, then $\Theta_{N, r_{N}} \triangle \Theta_{N, M} \cap \Gamma_{N}(\lambda) \neq \emptyset$. Thus, there are $u, v \in \Gamma_{N}(\lambda)$ such that $\min \left(M, r_{N}\right) \leq\|u-v\|_{2} \leq \max \left(M, r_{N}\right)$. For $N$ being so large that $r_{N}>r$ and $r_{N} \leq N / r$, this implies

$$
\max _{M: r \leq M \leq N / r}\left|\mathbb{E}\left[e^{-\left\langle\mu_{N, r_{N}}, f\right\rangle}\right]-\mathbb{E}\left[e^{-\left\langle\mu_{N, M}, f\right\rangle}\right]\right| \leq \mathbb{P}\left(\exists u, v \in \Gamma_{N}(\lambda): r \leq\|u-v\|_{2} \leq N / r\right),
$$

which by Fels and Hartung (2019, Theorem 2.2) tends to zero. This shows (3.3).

We set $M:=\min \left\{k: 2^{k}>r\right\}$. In light of Lemma 3.1, we work with $\mu_{N, M}$ instead of $\mu_{N, r_{N}}$. The reason for working with $M$ instead of $r_{N}$ is that it allows to consider the cluster on an $N$-independent scale, which, together with several approximations, eventually allows to reduce the proof of the full extremal process, $\mu_{N, r_{N}}$, to convergence of the extremal process that captures only the extreme local maxima, $\eta_{N, r_{N}}$. See also Remark 3.5 and (3.28), where it enters the proof. Suppose that the local maximum is taken at $v \in V_{N}$. We decompose into two fields. The idea is, for fixed $v \in V_{N}$, to use the Gibbs-Markov property of the underlying DGFF to write the field into independent components. One that captures the field inside $\Lambda_{M}(v)$ and another that captures the field outside, i.e. in $\Lambda_{M}^{c}(v) . v \in V_{N}$ later plays the role of a local maximum. Thus, we write

$$
\psi_{w}^{N}=\Phi_{w}^{M, v}+\tilde{\psi}_{w}^{\Lambda_{M}(v)}, \quad \text { for } w \in \Lambda_{M}(v)
$$

where

$$
\Phi_{w}^{M, v}:=\int_{0}^{1-\frac{\log M+\log _{+}\|v-w\|_{2}}{\log N}} \sigma(s) \nabla \phi_{w}^{N}(s) \mathrm{d} s+\int_{1-\frac{\log M+\log _{+}\|v-w\|_{2}}{\log N}}^{1} \sigma(s) \nabla \mathbb{E}\left[\phi_{w}^{N} \mid \sigma\left(\phi_{y}^{N}: y \in \partial[w]_{s} \cap \Lambda_{M}^{c}(v)\right)\right] \mathrm{d} s,
$$


and where

$$
\tilde{\psi}_{w}^{\Lambda_{M}(v)}=\int_{0}^{1} \sigma\left(1+(s-1) \frac{\log M+\log _{+}\|v-w\|_{2}}{\log N}\right) \phi_{w}^{\Lambda_{M}(v)}(s) \mathrm{d} s,
$$

and which we set equals zero on $\Lambda_{M}(v)^{c}$. The field in (3.6) encodes the increments when conditioning outside the local maximum $v \in V_{N}$ and its $M$-neighbourhood, $\Lambda_{M}(v)$. Note that it explicitely depends on $N$. The field in (3.7) encodes the remaining increments within $\Lambda_{M}(v)$. The following lemma points out the key idea behind the definitions in (3.6) and (3.7).

Lemma 3.2. Suppose $v \in V_{N}$ such that $\Lambda_{M}(v) \subset V_{N}$ and let $M=2^{k}$. Consider the sigma-algebra

$$
\mathcal{F}_{M, v}:=\sigma\left(\phi_{w}^{N}: w \in\{v\} \cup \Lambda_{M}(v)^{c}\right) .
$$

Then, for Lebesgue almost every $t \in \mathbb{R}$, and for any $A \in \mathcal{B}\left(\mathbb{R}^{\mathbb{Z}^{2}}\right)$,

$\mathbb{P}\left(\left(\psi_{v+w}^{N}-\Phi_{v+w}^{M, v}\right)_{w \in \mathbb{Z}^{2}} \in A \mid \mathcal{F}_{M, v}\right)=\mathbb{P}\left(\left(\tilde{\psi}_{v+w}^{\Lambda_{M}(v)}\right)_{w \in \mathbb{Z}^{2}} \in A \mid \tilde{\psi}_{v}^{\Lambda_{M}(v)}=t-\Phi_{v}^{M, v}\right)$, on $\left\{\psi_{v}^{N}=t\right\}$.

Proof: It is an immediate consequence using (3.5).

The following proposition is used to localize the initial increments, $\Phi_{v}^{M, v}$, of a local maximum at $v \in V_{N}$.

Proposition 3.3. Let $t \in \mathbb{R}$. There is $r_{0} \in \mathbb{N}$ such that, for any $\delta \in(0,1), r \geq r_{0}, N \in \mathbb{N}$, sufficiently large, $M \in(r, N / r)$ and $\gamma \in(0,1 / 2)$, there is a constant $C_{\delta}>0$, depending only on $\delta$,

$$
\begin{array}{r}
\mathbb{P}\left(\exists v \in V_{N}: \psi_{v}^{N} \geq m_{N}-t, \Phi_{v}^{M, v}-2 \log N \mathcal{I}_{\sigma^{2}}\left(1-\frac{\log M}{\log N}\right) \notin\left[-\log ^{\gamma}(M), \log ^{\gamma}(M)\right]\right) \\
\leq C_{\delta} e^{2 s} \sum_{k=\lfloor\log M\rfloor}^{\infty} k^{\frac{1}{2}-\gamma} \exp \left[-k^{\frac{2 \gamma-1}{2}}\right] .
\end{array}
$$

Proof: As in (3.5),

$$
\psi_{v}^{N}=\Phi_{v}^{M, v}+\tilde{\psi}_{v}^{\Lambda_{M}(v)},
$$

where the fields on the right hand side are independent. Using Fels and Hartung (2019, Lemma 3.1 (i)) for the first and the last field in (3.11), as well as by Green function asymptotics, see e.g. Biskup and Louidor $\left(2018,(3.47)\right.$, (B.5)), we deduce that, for any $\delta>0$, there is a constant $c_{\delta}>0$, such that

$$
\sup _{v \in V_{N}^{\delta}} \operatorname{Var}\left[\Phi_{v}^{M, v}\right] \leq 2 \log N \mathcal{I}_{\sigma^{2}}\left(1-\frac{\log M}{\log N}\right)+c_{\delta} .
$$

Moreover, $\left\{\Phi_{v}^{M, v}\right\}_{v \in V_{N}}$ is a centred Gaussian field. Thus, we can rerun the proof of Fels and Hartung (2019, Proposition 4.2), where the constant on the right of Fels and Hartung (2019, (4.13)) may now depend on $\delta$. This concludes the proof of Proposition 3.3.

The following lemma allows us to reduce the local field defined in (3.7) to a usual DGFF with a constant parameter.

Lemma 3.4. Let $v \in V_{N}^{\delta}$ and let $\left\{\tilde{\psi}_{w}^{\Lambda_{M}(v)}: w \in \Lambda_{M}(v)\right\}$ be the centred Gaussian field defined in (3.7). Then, for any $x, y \in \Lambda_{M}(v)$,

$$
\lim _{N \rightarrow \infty} \mathbb{E}\left[\tilde{\psi}_{x}^{\Lambda_{M}(v)} \tilde{\psi}_{y}^{\Lambda_{M}(v)}-\sigma^{2}(1) \phi_{x}^{\Lambda_{M}(v)} \phi_{y}^{\Lambda_{M}(v)}\right]=0 .
$$


In particular, $\left\{\tilde{\psi}_{w}^{\Lambda_{M}(v)}: w \in \Lambda_{M}(v)\right\}$ has asymptotically the same distribution as $\left\{\sigma(1) \phi_{w}^{\Lambda_{M}(v)}\right.$ : $\left.w \in \Lambda_{M}(v)\right\}$.

Proof: Note that for some $\epsilon>0$, by an Taylor expansion at 1 , we have $\sigma^{2}(1-s)=\sigma^{2}(1)-$ $\left(\sigma^{2}\right)^{\prime}(1)(1-s)+o\left(\left(\sigma^{2}\right)^{\prime}(1)(1-s)\right)$, for $s \in(1-\epsilon, 1]$. In particular, for any $v \in V_{N}$ and $x, y \in$ $\Lambda_{M}(v)$,

$$
\begin{aligned}
& \mathbb{E}\left[\tilde{\psi}_{x}^{\Lambda_{M}(v)} \tilde{\psi}_{y}^{\Lambda_{M}(v)}-\sigma^{2}(1) \phi_{x}^{\Lambda_{M}(v)} \phi_{y}^{\Lambda_{M}(v)}\right] \\
& \quad \leq 2 \int_{0}^{1}\left(\sigma^{2}\right)^{\prime}(1)(1-s) \frac{\log M+\log _{+}\|v-w\|_{2}}{\log N} \mathbb{E}\left[\nabla \phi_{x}^{\Lambda_{M}(v)}(s) \nabla \phi_{y}^{\Lambda_{M}(v)}(s)\right] \mathrm{d} s+o(1),
\end{aligned}
$$

where the right-hand side is of order $O\left(\frac{(\log M)^{2}}{\log N}\right)$ and tends to zero, as $N \rightarrow \infty$. This shows (3.13).

Remark 3.5. With regard to Lemma 3.2 and using Proposition 3.3, the cluster law around around a local maximum $v \in V_{N}^{\delta}$ can be written in the form

$$
\mathbb{P}\left(\left(\tilde{\psi}_{w}^{\Lambda_{M}(v)}\right)_{w \in \Lambda_{M}(v)} \in \cdot \mid \tilde{\psi}_{v}^{\Lambda_{M}(v)}=2 \log N\left(1-\mathcal{I}_{\sigma^{2}}\left(1-\frac{\log M}{\log N}\right)\right)+t, \tilde{\psi}_{w}^{\Lambda_{M}(v)} \leq \tilde{\psi}_{v}^{\Lambda_{M}(v)}\right) .
$$

Lemma 3.4 shows that (3.15) has the same weak limit, as $M \rightarrow \infty$ after $N \rightarrow \infty$, as the probability measure,

$\Xi^{(M, t)}(A):=\mathbb{P}\left(\sigma(1)\left(\phi_{0}^{\Lambda_{M}(0)}-\phi_{v}^{\Lambda_{M}(0)}\right)_{v \in \Lambda_{M}(0)} \in A \mid \phi_{0}^{\Lambda_{M}(0)}=2 \sigma(1) \log M+t, \phi^{\Lambda_{M}(0)} \leq \phi_{0}^{\Lambda_{M}(0)}\right)$,

with $A \in \mathcal{B}\left([0, \infty)^{\left|\Lambda_{M}(0)\right|}\right)$. It is crucial that (3.16) does not depend on $N$.

In the following lemma we show that the the cluster limit of the law $\Xi^{(M, t)}$ exists in a suitable sense. Recall the definition of the pinned field, $\left\{\phi_{v}^{\mathbb{Z}^{2} \backslash\{0\}}\right\}_{v \in \mathbb{Z}^{2} \backslash\{0\}}$, which is a centred Gaussian field with covariances given in (1.17) and whose value at the origin is pinned to zero.

Lemma 3.6. Fix $r, j \geq 1$ and let $c_{1} \in(0, \infty)$. For $M=\min \left\{k: 2^{k}>r\right\}$, uniformly in $f \in C_{c}\left(\overline{\mathbb{R}}^{\Lambda_{j}}\right)$ and $t=o(\log M)$,

$$
\lim _{M \rightarrow \infty} \int_{\mathbb{R}^{\mathbb{Z}^{2}}} \Xi^{(M, t)}(\mathrm{d} \theta) f(\theta)=\int_{\mathbb{R}^{\mathbb{Z}^{2}}} \Xi(\mathrm{d} \theta) f(\theta)
$$

where $\Xi(\cdot):=\lim _{r \rightarrow \infty} \Xi_{r}(\cdot)$, and where $\Xi_{r}(A)$, for $A \in \mathcal{B}\left([0, \infty)^{\mathbb{Z}^{2}}\right)$, is given by

$$
\mathbb{P}\left(\left(\phi_{v}^{\mathbb{Z}^{2} \backslash\{0\}}+2 \sigma(1) \mathfrak{a}(v)\right)_{v \in \mathbb{Z}^{2}} \in A \mid \phi_{v}^{\mathbb{Z}^{2} \backslash\{0\}}+2 \sigma(1) \mathfrak{a}(v) \geq 0:\|v\|_{2} \leq r\right),
$$

and $\mathfrak{a}$ being the potential kernel as in (1.16).

Proof: Convergence of the finite dimensional distributions of the measures $\Xi_{r}(\cdot)$ is a simple consequence of the DGFF satisfying the strong FKG-inequality (see Biskup and Louidor, 2018, Lemma B.8 and Grimmett, 1999, Theorem (2.4)), which implies that $r \mapsto \theta_{r}$ is stochastically increasing. Thus, $\lim _{r \rightarrow \infty} \Xi_{r}(A)$ exists for any event $A$, depending on only a finite number of coordinates. Next, 
we prove that $\left\{\Xi_{r}\right\}_{r}$ is tight, which then implies that $\Xi$ is a distribution on $\mathbb{R}^{\mathbb{Z}^{2}}$. By a union and a Gaussian tail bound, for any $r \geq k_{0}>0$, there are constants $C, \tilde{C}>0$ such that

$$
\begin{aligned}
& \mathbb{P}\left(\exists v, k_{0} \leq\|v\|_{2} \leq r: \phi_{v}^{\mathbb{Z}^{2} \backslash\{0\}}>2 \sigma(1) \log \|v\|_{2}\right) \\
& \leq \sum_{k=k_{0}}^{r} 4 k \mathbb{P}\left(\sup _{\|v\|_{\infty}=k} \phi_{v}^{\mathbb{Z}^{2} \backslash\{0\}}>2 \sigma(1) \log k+\frac{1}{2} \log (2)\right) \\
& \leq C \sum_{k=k_{0}}^{r} \frac{4 k}{\sqrt{\log k}} \exp \left[-\sigma^{2}(1) \log k+c_{0}\right] \leq \tilde{C} \sum_{k=k_{0}}^{\infty} \frac{1}{\sqrt{\log k}} \exp \left[-\left[\sigma^{2}(1)-1\right] \log k\right] .
\end{aligned}
$$

As the sum converges and vanishes, as $k_{0} \rightarrow \infty$, we deduce tightness of $\left(\Xi_{r}\right)_{r \in \mathbb{N}}$ and so $\Xi\left(\mathbb{R}^{\mathbb{Z}^{2}}\right)=1$. In the last step, we show that it takes the particular form as in (3.18). Note that by the GibbsMarkov property of the DGFF (Biskup and Louidor, 2018, Lemma B.6) we have, for $v \in \Lambda_{M}(0)$,

$$
\phi_{v}^{\Lambda_{M}(0)}=\phi_{v}^{\Lambda_{M}(0) \backslash\{0\}}+g_{M}(v) \phi_{0}^{\Lambda_{M}(0)},
$$

where $g_{M}(x)$ is discrete harmonic with $g_{M}(0)=1$ and $g_{M}(x)=0$, for $x \notin \Lambda_{M}(0)$, and equality holds in distribution. Thus, we have that $\phi_{v}^{\Lambda_{M}(0)}$ conditioned on $\phi_{0}^{\Lambda_{M}(0)}=2 \sigma(1) \log M+t$ shifts the mean of $\phi_{0}^{\Lambda_{M}(0)}-\phi_{v}^{\Lambda_{M}(0)}$ by

$$
(2 \sigma(1) \log M+t)\left(1-g_{M}(v)\right) .
$$

Note that by Biskup and Louidor (2018, (3.47))

$$
1-g_{M}(v)=\frac{G_{V_{M}}((M / 2, M / 2),(M / 2, M / 2))-G_{V_{M}}((M / 2, M / 2),(M / 2, M / 2)+v)}{G_{V_{M}}((M / 2, M / 2),(M / 2, M / 2))}, \quad v \in \Lambda_{M}(0),
$$

with $G_{V_{M}}$ being the Green's function of the simple random walk on $V_{M}$ as in (1.1). Thus, together with the representation of the potential kernel, $\mathfrak{a}$, as in (1.16), and using that Proposition 3.3 allows to assume, $t=o(\sqrt{\log M})$, we deduce that (3.21) is asymptotically equal to $2 \sigma(1) \mathfrak{a}(v)$, as $M \rightarrow \infty$. In particular, the law of $v \mapsto \phi_{0}^{\Lambda_{M}(0)}-\phi_{v}^{\Lambda_{M}(0)}$ conditioned on $\phi_{0}^{\Lambda_{M}(0)}=2 \sigma(1) \log M$ converges, as $M \rightarrow \infty$, in the sense of finite dimensional distributions to

$$
\phi_{v}^{\mathbb{Z}^{2} \backslash\{0\}}+2 \sigma(1) \mathfrak{a}(v), \quad v \in \mathbb{Z}^{2} .
$$

This concludes the proof of Lemma 3.6.

Having weak convergence of the auxiliary cluster law, $\nu_{r}$, we are now in a position to prove convergence of the full extremal process.

Proof of Theorem 1.6: First note that by Lemma 3.1 we can work with $M=\min \left\{k: 2^{k}>r_{N}\right\}$ instead of $r_{N}$. Let $f:[0,1]^{2} \times \mathbb{R} \times \overline{\mathbb{R}}^{\mathbb{Z}^{2}} \mapsto[0, \infty)$ be a continuous function with compact support. In addition, assume that, for any $x \in[0,1]^{2}$ and $t \in \mathbb{R}, f(x, t, \theta)$ depends only on $\left\{\theta_{y}: y \in \Lambda_{M}(x)\right\}$ and further, that $f$ vanishes if $t, \max _{y \in \Lambda_{M}(0)}\left|\theta_{y}\right|>h$, for some $h>0$. Let $V_{N}=\cup_{i=1}^{(N / M)^{2}} V_{M, i}$ be a decomposition of $V_{N}$ into disjoint shifts of $V_{M}$. Recall $\Theta_{N, M}=\left\{v \in V_{N}: \psi_{v}^{N}=\max _{u \in \Lambda_{M}(v)} \psi_{u}^{N}\right\}$, the set of $M$-local maxima. Moreover, let $\delta \in(0,1)$ and set

$$
\mu_{N, M, \delta}:=\sum_{v \in \cup_{i=1}^{(N / M)^{2}} V_{M, i}^{\delta}} \mathbb{1}_{v \in \Theta_{N, M}} \delta_{v / N} \otimes \delta_{\psi_{v}^{N}-m_{N}} \otimes \delta_{\left\{\psi_{v}^{N}-\psi_{v+w}^{N}: w \in \mathbb{Z}^{2}\right\}}
$$


By Proposition 3.3, Fels and Hartung (2019, Proposition 5.1) and Fels and Hartung (2019, Theorem 2.2), it suffices to compute

$$
\begin{aligned}
\lim _{\delta \rightarrow 0} \lim _{M \rightarrow \infty} \lim _{N \rightarrow \infty} \mathbb{E}[ & e^{-\left\langle\mu_{N, M, \delta}, f\right\rangle} \mathbb{1}_{\|v-w\|_{2}>4 M: v, w \in \Theta_{N, M}} \\
& \left.\times \mathbb{1}_{\left\{\Phi_{v}^{N, v}-2 \log N \mathcal{I}_{\sigma^{2}}\left(1-\frac{\log M}{\log N}\right) \in\left[-\log ^{\gamma}(M), \log ^{\gamma}(M)\right]: v \in \Theta_{N, M}\right\}}\right] .
\end{aligned}
$$

Set

$$
\begin{aligned}
& f_{N, M}(v / N, t):= \\
& -\log \mathbb{E}\left[\exp \left[-f\left(x, t,\left(\psi_{v}^{N}-\Phi_{v}^{M, v}-\psi_{v+w}^{N}+\Phi_{v+w}^{M, v}: w \in \mathbb{Z}^{2}\right)\right)\right] \mid \psi_{v}^{N}=m_{N}+t, v \in \Theta_{N, M}\right] .
\end{aligned}
$$

Conditioning on position, $x_{i} N$, and height, $m_{N}+t_{i}$, of local maxima in $\cup_{i=1}^{(N / M)^{2}} V_{M, i}^{\delta}$ and on the sigma-algebra $\sigma\left(\phi_{w}^{N}: w \in \cup \partial \Lambda_{M}\left(x_{i} N\right)\right)$, using Lemma 3.2 and the Taylor approximation for the cluster process as in the proof of Lemma 3.4, we can rewrite (3.25) as

$$
\begin{aligned}
& \mathbb{E}\left[\prod_{i=1}^{(N / M)^{2}} e^{-f_{N, M}\left(x_{i}, t_{i}\right)} \mathbb{1}_{N\left\|x_{j}-x_{k}\right\|_{2}>4 M: x_{j} N, x_{k} N \in \Theta_{N, M}}\right. \\
&\left.\times \mathbb{1}_{\left\{\Phi_{v}^{N, v}-2 \log N \mathcal{I}_{\sigma^{2}}\left(1-\frac{\log M}{\log N} \in\left[-\log ^{\gamma}(M), \log ^{\gamma}(M)\right]\right): v \in \Theta_{N, M}\right\}}\right] .
\end{aligned}
$$

On $\left\{\Phi_{v}^{N, v}-2 \log N \mathcal{I}_{\sigma^{2}}\left(1-\frac{\log M}{\log N}\right) \in\left[-\log ^{\gamma}(M), \log ^{\gamma}(M)\right]: v \in \Theta_{N, M}\right\}$, Lemma 3.2, Lemma 3.4, Remark 3.5 and Lemma 3.6 imply

$$
\lim _{M \rightarrow \infty} \lim _{N \rightarrow \infty} f_{N, M}(x, t)=f_{\Xi}(x, t):=-\log \int_{[0, \infty)^{\mathbb{Z}^{2}}} e^{-f(x, t, \theta)} \Xi(\mathrm{d} \theta) .
$$

In particular, the convergence in (3.28) is uniform in $x \in \cup_{i=1}^{(N / M)^{2}} V_{M, i}^{\delta}$ and $t \in \mathbb{R}$. Moreover, note that thanks to the localization in Proposition 3.3 the right-hand side of (3.28) does not depend on $N$, which allows us in the following step to use Theorem 1.4, i.e. using (3.27) and Proposition 3.3, we can rewrite $(3.25)$ as

$$
\mathbb{E}\left[e^{-\left\langle\eta_{N, M}, f_{\Xi}\right\rangle}\right]+o(1)
$$

Applying Theorem 1.4 to (3.29), we obtain

$$
\begin{aligned}
& \lim _{M \rightarrow \infty} \lim _{N \rightarrow \infty} \mathbb{E}\left[e^{-\left\langle\mu_{N, M}, f_{\Xi}\right\rangle}\right] \\
& =\mathbb{E}\left[\exp \left[-\int_{[0,1]^{2} \times \mathbb{R}} Y(\mathrm{~d} x) \otimes \beta e^{-2 h} \mathrm{~d} h\left(1-e^{-f_{\Xi}(x, h)}\right)\right]\right] \\
& =\mathbb{E}\left[\exp \left[-\int_{[0,1]^{2} \times \mathbb{R} \times \mathbb{R}^{\mathbb{Z}^{2}}} Y(\mathrm{~d} x) \otimes \beta e^{-2 h} \mathrm{~d} h \otimes \Xi(\mathrm{d} \theta)\left(1-e^{-f(x, h, \theta)}\right)\right]\right] .
\end{aligned}
$$

Noting that the last line in (3.30) is the Laplace transform of a Poisson point process with intensity $Y(\mathrm{~d} x) \otimes \beta e^{-2 h} \mathrm{~d} h \otimes \Xi(\mathrm{d} \theta)$, concludes the proof.

\section{Proof of Theorem 2.5}

As the proof of Theorem 2.5 is quite involved, we start by explaining the principal idea and outlining the main steps. 
Step 1: 3 Field approximation. The first step consists in reducing the proof the maximum over finitely many disjoint subsets of the original field to prove the same for an auxiliary Gaussian field that is structurally simpler and which we have successfully used in Fels and Hartung (2019) in order to prove convergence in law of the global maximum. This is the so-called 3-field approximation which we recall in Subsection 4.1. Its main advantage lies in the fact that it decouples macroscopic and microscopic scales. The reason why such a simplification should be possible is due to a localization of paths of extremal particles, similarly as in Proposition 3.3, as well as the macroscopic separation of extreme local maxima, as stated in (1.7). The latter implies that extreme local maxima are at mutual distance of $O(N)$, and thus, their correlations are of $O(1)$. In particular, all extremal points belong to $O(1)$ neighbourhoods of such extreme local maxima. Moreover, the large scale increments for vertices in a $O(1)$ neighbourhood of such an extreme local maximum are essentially the same for all vertices in one such neighbourhood.

Step 2: Gaussian comparison. In Subsection 4.2, we show, using Theorem 5.2, a vector version of Slepian's lemma, that it suffices to prove convergence in law for a finite number of extreme local maxima of the auxiliary 3 -field.

Step 3: Convergence of the extreme local maxima of the auxiliary field. We prove convergence of the extreme local maxima of the auxiliary field in Subsection 4.3. The proof is divided into two steps.

Step 3.1: Coupling. First, we couple the 3-field approximation to independent random variables. The crucial point here is that these do not depend on $N$, and that they are asymptotically equal in distribution to the extreme local centred maxima of the 3-field approximation. A key ingredient is the localization of the paths of extremal vertices at coarse scales which was obtained in Fels and Hartung (2019, Lemma 5.7). A second important ingredient are the precise right-tail asymptotics for the maximum over sub-boxes. Such right-tail asymptotics are proven in Fels and Hartung (2019, Proposition 5.8). Next, we prove existence of the limiting law of the extreme local maxima using the coupling described above. First, we prove tightness of finitely many centred extreme local maxima of the scale-inhomogeneous DGFF. Next, we use the Gaussian comparison arguments in Subsection 4.2, to obtain tightness for the 3-field approximation. Tightness in combination with the coupling allows to deduce convergence in law of the auxiliary process, which is the content of Theorem 4.7.

Step 3.2: Identification of the limiting law. Next, we identify the limit law of extreme local maxima. We show that it is given as the Laplace transform of the intensity measure, $Y$, on the disjoint subsets. This also implies uniqueness of the intensity measure, $Y$, in Theorem 2.4.

4.1. 3-field approximation. We first decompose the underlying grid $V_{N}$. Assume $N=2^{n}$ to be much larger than any other forthcoming integers. Next, pick two large integers $L=2^{l}$ and $K=2^{k}$. Partition $V_{N}$ in a disjoint union of $(K L)^{2}$ boxes, $\mathcal{B}_{N / K L}=\left\{B_{N / K L, i}: i=1, \ldots,(K L)^{2}\right\}$, each of side length $N / K L$. Let $v_{N / K L, i} \in V_{N}$ be the left bottom corner of box $B_{N / K L, i}$ and write $w_{i}=\frac{v_{N / K L, i}}{N / K L}$. We consider $\left\{w_{i}\right\}_{i=1, \ldots,(K L)^{2}}$ as the vertices of a box $V_{K L}$. Analogously, let $K^{\prime}=2^{k^{\prime}}$ and $L^{\prime}=2^{l^{\prime}}$ be two integers, such that $K^{\prime} L^{\prime}$ divides $N$. Let $\mathcal{B}_{K^{\prime} L^{\prime}}=\left\{B_{K^{\prime} L^{\prime}, i}: i=1, \ldots,\left[N /\left(K^{\prime} L^{\prime}\right)\right]^{2}\right\}$ be a disjoint partitioning of $V_{N}$ with boxes $B_{K^{\prime} L^{\prime}, j}$, each of side length $K^{\prime} L^{\prime}$. The left bottom corner of a box $B_{K^{\prime} L^{\prime}, i}$ we call $v_{K^{\prime} L^{\prime}, i}$.

We take limits in the order $N, K^{\prime}, L^{\prime}, K$ and finally $L$, for which we write $\left(L, K, L^{\prime}, K^{\prime}, N\right) \Rightarrow \infty$. The macroscopic or coarse field, $\left\{S_{v}^{N, c}\right\}_{v \in V_{N}}$, is a centred Gaussian field with covariance matrix $\Sigma^{c}$, 
with entries given by

$$
\Sigma_{u, v}^{c}:=\sigma^{2}(0) \mathbb{E}\left[\phi_{w_{i}}^{K L} \phi_{w_{j}}^{K L}\right], \quad \text { for } u \in B_{N / K L, i}, v \in B_{N / K L, j},
$$

where $\left\{\phi_{v}^{K L}\right\}_{v \in V_{K L}}$ is a DGFF on $V_{K L}$. It captures the macroscopic dependence. The microscopic or "bottom field", $\left\{S_{v}^{N, b}\right\}_{v \in V_{N}}$, is a centred Gaussian field with covariance matrix $\Sigma^{b}$ defined entry-wise as

$$
\Sigma_{u, v}^{b}:= \begin{cases}\sigma^{2}(1) \mathbb{E}\left[\phi_{u-v_{K^{\prime} L^{\prime}, i}}^{K^{\prime} L^{\prime}} \phi_{v-v_{K^{\prime} L^{\prime}, i}}^{K^{\prime} L^{\prime}}\right], & \text { if } u, v \in B_{K^{\prime} L^{\prime}, i} \\ 0, & \text { else, }\end{cases}
$$

where $\left\{\phi_{v}^{K^{\prime} L^{\prime}}\right\}_{v \in V_{K^{\prime} L^{\prime}}}$ is a DGFF on $V_{K^{\prime} L^{\prime}}$. It captures "local" correlations. The third centred Gaussian field, $\left\{S_{v}^{N, m}\right\}_{v \in V_{N}}$, approximates the "intermediate" scales. It is a modified inhomogeneous branching random walk, defined pointwise as

$$
S_{v}^{N, m}:=\sum_{j=k^{\prime}+l^{\prime}}^{n-l-k} \sum_{B \in \mathcal{B}_{j}\left(v_{K^{\prime} L^{\prime}, i^{\prime}}\right)} 2^{-j} \sqrt{\log 2} b_{i, j, B}^{N} \int_{n-j-1}^{n-j} \sigma\left(\frac{s}{n}\right) \mathrm{d} s, \quad \text { for } v \in B_{N / K L, i} \cap B_{K^{\prime} L^{\prime}, i^{\prime}},
$$

with $\left\{b_{i, j, B}^{N}: B \in \cup_{i^{\prime}} \mathcal{B}_{j}\left(v_{K^{\prime} L^{\prime}, i^{\prime}}\right), i=1, \ldots,(K L)^{2}, j=1, \ldots,\left(N / K^{\prime} L^{\prime}\right)^{2},\right\}$ being a family of independent standard Gaussian random variables and where $\mathcal{B}_{j}\left(v_{K^{\prime} L^{\prime}, i^{\prime}}\right)$ is the collection of boxes, $B \subset V_{N}$, of side length $2^{j}$ and lower left corner in $V_{N}$, that contain the element $v_{K^{\prime} L^{\prime}, i^{\prime}}$. In order to avoid boundary effects, we restrict our considerations onto a slightly smaller set, which is defined next. Consider the disjoint union of $N / L$ - and $L$-boxes, that is $\mathcal{B}_{N / L}=\left\{B_{N / L, i}: i=1 \ldots, L^{2}\right\}$ and $\mathcal{B}_{L}=\left\{B_{L, i}: i=1, \ldots,(N / L)^{2}\right\}$. Analogously, let $v_{N / L, i}$ and $v_{L, i}$ be the bottom left corners of the boxes $B_{N / L, i}, B_{L, i}$ containing $v$. For a box $B$, let $B^{\delta} \subset B$ be the set $B^{\delta}=\{v \in B$ : $\left.\min _{z \in \partial B}\|v-z\| \geq \delta l_{B}\right\}$, where $l_{B}$ denotes the side length of the box $B$. Finally, let

$$
V_{N, \delta}^{*}:=\left\{\cup_{1 \leq i \leq L^{2}} B_{N / L, i}^{\delta}\right\} \cap\left\{\cup_{1 \leq i \leq(K L)^{2}} B_{N / K L, i}^{\delta}\right\} \cap\left\{\cup_{1 \leq i \leq(N / L)^{2}} B_{L, i}^{\delta}\right\} \cap\left\{\underset{1 \leq i \leq(N / K L)^{2}}{\cup} B_{K L, i}^{\delta}\right\} .
$$

The next lemma ensures that the sum of the three fields, $\left\{S_{v}^{N, c}\right\}_{v \in V_{N}},\left\{S_{v}^{N, m}\right\}_{v \in V_{N}},\left\{S_{v}^{N, b}\right\}_{v \in V_{N}}$, approximates well the scale-inhomogeneous DGFF, $\left\{\psi_{v}^{N}\right\}_{v \in V_{N}}$.

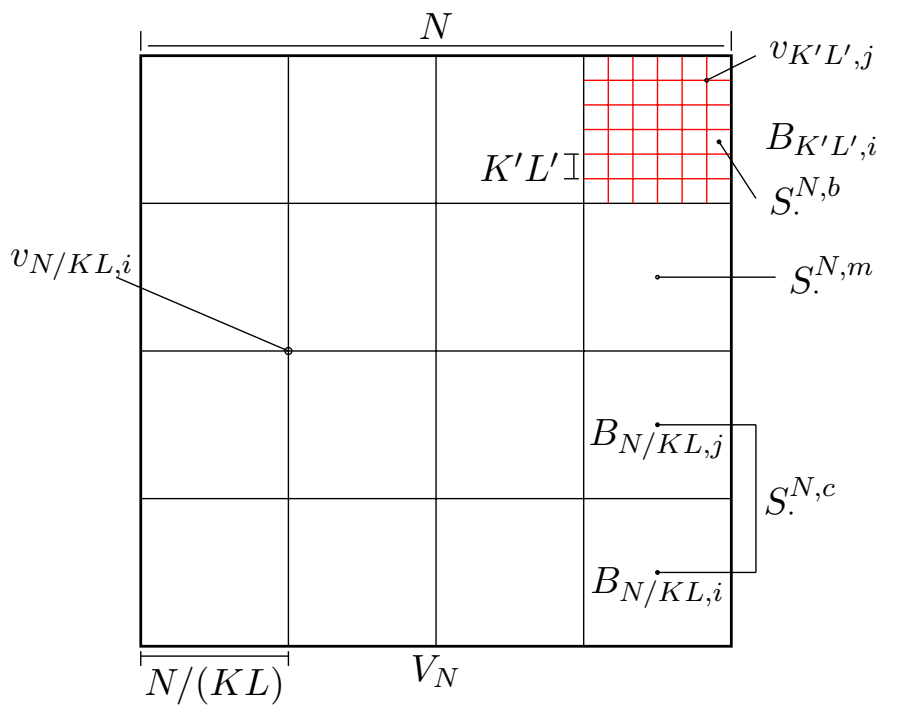

Figure 1: 3-field decomposition 
Lemma 4.1. Fels and Hartung (2019, Lemma 5.2, Lemma 5.3) There are non-negative uniformly bounded sequences of constants $a_{K^{\prime} L^{\prime}, \bar{v}}$ and a family of i.i.d. Gaussians $\left\{\Theta_{j}\right\}_{j=1, \ldots,\left(N / K^{\prime} L^{\prime}\right)^{2}}$, such that, for $v \in B_{K^{\prime} L^{\prime}, j}, v \equiv \bar{v} \bmod K^{\prime} L^{\prime}$, i.e. $\bar{v}=v-v_{K^{\prime} L^{\prime}, j}$, and when setting

$$
S_{v}^{N}:=S_{v}^{N, c}+S_{v}^{N, m}+S_{v}^{N, b}+a_{K^{\prime}, L^{\prime}, j} \Theta_{j},
$$

we have

$$
\limsup _{\left(L, K, L^{\prime}, K^{\prime}, N\right) \Rightarrow \infty}\left|\operatorname{Var}\left(S_{v}^{N}\right)-\operatorname{Var}\left(\psi_{v}^{N}\right)-4 \alpha\right|=0
$$

for some $\alpha>0$. Further, there exists a sequence $\left\{\epsilon_{N, K L, K^{\prime} L^{\prime}}^{\prime} \geq 0\right\}$ with $\limsup _{\left(L, K, L^{\prime}, K^{\prime}, N\right) \Rightarrow \infty} \epsilon_{N, K L, K^{\prime} L^{\prime}}^{\prime}=$ 0 and bounded constants $C_{\delta}, C>0$, such that for all $u, v \in V_{N, \delta}^{*}$ :

(1) If $u, v \in B_{L^{\prime}, i}$, then $\left|\mathbb{E}\left[\left(S_{u}^{N}-S_{v}^{N}\right)^{2}\right]-\mathbb{E}\left[\left(\psi_{u}^{N}-\psi_{v}^{N}\right)^{2}\right]\right| \leq \epsilon_{N, K L, K^{\prime} L^{\prime}}^{\prime}$

(2) If $u \in B_{N / L, i}, v \in B_{N / L, j}$ with $i \neq j$, then $\left|\mathbb{E}\left[S_{u}^{N} S_{v}^{N}\right]-\mathbb{E}\left[\psi_{u}^{N} \psi_{v}^{N}\right]\right| \leq \epsilon_{N, K L, K^{\prime} L^{\prime}}^{\prime}$

(3) In all other cases, that is if $u, v \in B_{N / L, i}$ but $u \in B_{L^{\prime}, i^{\prime}}$ and $v \in B_{L^{\prime}, j^{\prime}}$ for some $i^{\prime} \neq j^{\prime}$, it holds that $\left|\mathbb{E}\left[S_{u}^{N} S_{v}^{N}\right]-\mathbb{E}\left[\psi_{u}^{N} \psi_{v}^{N}\right]\right| \leq C_{\delta}+40 \alpha$.

The field, $\left\{S_{v}^{N}\right\}_{v \in V_{N}}$, defined in (4.5) is the approximating 3 -field we work with. The point in choosing two parameters, $K$ and $L$, for boxes of size $N / K L$ instead of choosing just one, is the fact that this allows to show that at macroscopic scales, that is for vertices $v \in B_{N / L, i}$ and $w \in B_{N / L, j}$ for $i \neq j$, covariances of the actual and the approximating field asymptotically match, when taking limits $N \rightarrow \infty$ followed by $K \rightarrow \infty$, as stated in (2) of Lemma 4.1. Similiarly, the choice of two paremeters, $K^{\prime}$ and $L^{\prime}$, instead of just a single one, allows to deduce that the approximating field at fine scales, for $v, w \in B_{L^{\prime}, i}$ in the same box of side length $L^{\prime}$, has asymptotically the same correlation structure as the original field, as $N \rightarrow \infty$ followed by $K^{\prime} \rightarrow \infty$, as follows from (1) and (4.6) in Lemma 4.1. These two facts are of crucial importance when using Gaussian comparison in the following Subsection 4.2, in which we show that it suffices to prove convergence of extreme local maxima of the auxiliary 3 -field.

4.2. Reduction to approximating field. In the following, we generalize the approximation results from Fels and Hartung (2019) to the case of countably many local maxima. We show that extreme local maxima of $\left\{\psi_{v}^{N}\right\}_{v \in V_{N}}$ are well approximated by those of $\left\{S_{v}^{N}\right\}_{v \in V_{N}}$. As we need to compare probability measures on $\mathbb{R}^{p}$, we use the Lévy-Prokhorov metric $d(\cdot, \cdot)$, to measure distances between probability measures on $\mathbb{R}^{p}$. For two probability measures, $\mu$ and $\nu$, it is given by

$$
d(\mu, \nu)=\inf \left\{\delta>0: \mu(B) \leq \nu\left(B^{\delta}\right)+\delta \text { for all open sets } B\right\},
$$

where $B^{\delta}=\left\{y \in \mathbb{R}^{p}:|x-y|<\delta\right.$, for some $\left.x \in B\right\}$. Further, let

$$
\begin{aligned}
\tilde{d}(\mu, \nu) & =\inf \left\{\delta>0: \mu\left(\left(x_{1}, \infty\right), \ldots,\left(x_{p}, \infty\right)\right)\right. \\
& \left.\leq \nu\left(\left(x_{1}-\delta, \infty\right), \ldots,\left(x_{p}-\delta, \infty\right)\right)+\delta, \forall\left(x_{1}, \ldots, x_{p}\right) \in \mathbb{R}^{p}\right\},
\end{aligned}
$$

which is a measure for stochastic domination. In particular, if $\tilde{d}(\mu, \nu)=0$, then $\nu$ stochastically dominates $\mu$. Note, unlike $d(\cdot, \cdot), \tilde{d}(\cdot, \cdot)$ is not symmetric. Abusing notation, we write for random vector $X, Y$ with laws $\mu_{X}, \mu_{Y}, d(X, Y)$ instead of $d\left(\mu_{X}, \mu_{Y}\right)$ and likewise for $\tilde{d}$. Fix $r \in \mathbb{N}$ and let $\mathcal{B}_{r}$ of $V_{\lfloor N / r\rfloor r}$ into sub-boxes of side length $r$. Let $\mathcal{B}=\cup_{r \in \mathbb{N}, r \leq N} \mathcal{B}_{r}$ and $\left\{g_{b}\right\}_{B \in \mathcal{B}}$ be a collection of i.i.d. standard Gaussian random variables. For $v \in V_{N}$, denote by $B_{r}(v) \in \mathcal{B}_{r}$ the box containing $v$. For $r_{1}, r_{2} \in \mathbb{N}, r_{1}, r_{2} \leq N, A \subset[0,1]^{2}, \tilde{\sigma}_{1}, \tilde{\sigma}_{2} \in \mathbb{R}_{+}$, we write

$$
\bar{\psi}_{v}^{N}:=\psi_{v}^{N}+\tilde{\sigma}_{1} g_{B_{v, r_{1}}}+\tilde{\sigma}_{2} g_{B_{v, N / r_{2}}},
$$


and for a general field $\left\{g_{v}^{N}\right\}_{v \in V_{N}}$,

$$
g_{N, A}^{*}:=\max _{v \in V_{N}: v / N \in A} g_{v}^{N} .
$$

Fix $p \in \mathbb{N}$ and disjoint, open, non-empty, simply connected sets $A_{1}, \ldots, A_{p} \subset[0,1]^{2}$. The key result in this sub-section is the following.

Lemma 4.2. Let $p \in \mathbb{N}$, and $A_{1}, \ldots, A_{p} \subset[0,1]^{2}$ be disjoint, open and non-empty. Then,

$$
\limsup _{\left(L, K, L^{\prime}, K^{\prime}, N\right) \Rightarrow \infty} d\left(\left(\psi_{N, A_{i}}^{*}-m_{N}\right)_{1 \leq i \leq p},\left(S_{N, A_{i}}^{*}-m_{N}-4 \alpha\right)_{1 \leq i \leq p}\right)=0 .
$$

Lemma 4.2 reduces the proof of convergence in law of multiple extreme local maxima of the scaleinhomogeneous DGFF to the structurally simpler field, $\left\{S_{v}^{N}\right\}_{v \in V_{N}}$, as microscopic and macroscopic dependence are decoupled per definition. A key simplification for its analysis is the fact that the coarse field, $\left\{S_{v}^{N, c}\right\}_{v \in V_{N}}$, is constant over large boxes, $B \in \mathcal{B}_{N / K L}$. This avoids the necessity to control both position and height of the local maxima of the fine field, $\left\{S_{v}^{N, f}=S_{v}^{N}-S_{v}^{N, c}\right\}_{v \in V_{N}}$.

Proof of Lemma 4.2: We refrain from giving a complete proof here as the proof of Lemma 4.2 follows in complete analogy to the proof of Fels and Hartung (2019, Lemma 5.4). We instead explain the overall idea and provide the necessary generalized statements in the remaining subsection. The general idea is to use Gaussian comparison to use Lemma 4.1, i.e. the fact that the covariance structure of the scale-inhomogeneous DGFF and the approximating Gaussian field, $\left\{S_{v}^{N}\right\}_{v \in V_{N}}$, are very close. However, such an application is not straightforward as variances do not match and as one lacks domination of the covariances of one field by the covariances of the other. We address this issue using the following simple observation: In Lemma 4.3 we show that small Gaussian perturbations of the scale-inhomogeneous DGFF at microscopic and macroscopic scales result in a deterministic shift of its extreme local maxima. The proof of this goes in two steps. First, one can use Gaussian interpolation to write the scale-inhomogeneous DGFF as the weighted sum of two such fields, where the weights are chosen in such a way that one of the fields carries almost the entire variance. This allows to relate extreme local maxima of the original field with extreme local maxima of the field with the larger variances with an additional explicit random shift. We then show in in a second step in Proposition 4.6 that it is possible to choose explicit perturbations that live only on a macroscopic or microscopic scale. In the second key step in the proof Lemma 4.2 one uses Gaussian comparison to show asymptotic stochastic domination of extreme local maxima, provided that, in the limit, variances match and covariances of one of the field dominate the covariances of the other. This is the contents of Lemma 4.5. To conclude the proof, we combine this with the first step. Choosing the size of the perturbations of the scale-inhomogeneous DGFF appropriately together with Lemma 4.1 allows to apply Lemma 4.5 to show stochastic domination in both directions when comparing extreme local maxima of suitably perturbed scale-inhomogeneous DGFFs with those of the auxiliary Gaussian field, $\left\{S_{v}^{N}\right\}_{v \in V_{N}}$. In particular, as by Lemma 4.1 one can choose the perturbations to match asymptotically, this implies that extreme local maxima of the field, $\left\{S_{v}^{N}\right\}_{v \in V_{N}}$, share, in the limit, the same law as those of perturbed scale-inhomogeneous DGFFs. Lemma 4.3 then relates their law to the law of extreme local maxima of the original scale-inhomogeneous DGFF, up to a deterministic shift.

In the remaining part of this subsection, we provide the missing ingredients used in the proof of Lemma 4.2.

Lemma 4.3. For $\tilde{\sigma}=\left(\tilde{\sigma}_{1}, \tilde{\sigma}_{2}\right) \in \mathbb{R}_{+}^{2}$, it holds

$$
\limsup _{r_{1}, r_{2} \rightarrow \infty} \limsup _{N \rightarrow \infty} d\left(\left(\psi_{N, A_{i}}^{*}-m_{N}\right)_{1 \leq i \leq p},\left(\bar{\psi}_{N, A_{i}}^{*}-m_{N}-\|\tilde{\sigma}\|_{2}^{2}\right)_{1 \leq i \leq p}\right)=0
$$


where $\left\{\bar{\psi}_{v}^{N}\right\}_{v \in V_{N}}$ is as defined in (4.9).

For the proof of Lemma 4.3 we need some additional estimates.

Lemma 4.4. Let $\left\{\bar{\psi}_{v}^{N}\right\}_{v \in V_{N}}$ be a centred Gaussian field and $c>0$ a constant, such that, for any $v, w \in V_{N},\left|\mathbb{E}\left[\bar{\psi}_{v}^{N} \bar{\psi}_{w}^{N}\right]-\mathbb{E}\left[\psi_{v}^{N} \psi_{w}^{N}\right]\right| \leq c$. Moreover, let $A \subset[0,1]^{2}$ be an open, non-empty subset and $\left\{g_{v}^{N}\right\}_{v \in V_{N}}$ be a collection of independent random variables, such that

$$
\mathbb{P}\left(g_{v}^{N} \geq 1+y\right) \leq e^{-y^{2}} \quad \text { for } v \in V_{N} .
$$

Then, there is a constant $C=C(\alpha)>0$ such that, for any $\epsilon>0, N \in \mathbb{N}$ and $x \geq-\epsilon^{1 / 2}$,

$$
\mathbb{P}\left(\max _{v \in V_{N}: v / N \in A}\left(\bar{\psi}_{v}^{N}+\epsilon g_{v}^{N}\right) \geq m_{N}+x\right) \leq \mathbb{P}\left(\max _{v \in V_{N}: v / N \in A} \bar{\psi}_{v}^{N} \geq m_{N}+x-\sqrt{\epsilon}\right)\left(C e^{-C^{-1} \epsilon^{-1}}\right) .
$$

Proof: Set $\Gamma_{y}:=\left\{v \in V_{N}: v / N \in A, y / 2 \leq \epsilon g_{v}^{N} \leq y\right\}$. Then, by conditioning on $\Gamma_{2^{i} \sqrt{\epsilon}}$,

$$
\begin{aligned}
\mathbb{P}\left(\max _{v \in V_{N}: v / N \in A}\left(\bar{\psi}_{v}^{N}+\epsilon g_{v}^{N}\right) \geq m_{N}+x\right) \leq & \mathbb{P}\left(\max _{v \in V_{N}: v / N \in A} \bar{\psi}_{v}^{N} \geq m_{N}+x-\sqrt{\epsilon}\right) \\
& +\sum_{i=0}^{\infty} \mathbb{E}\left[\mathbb{P}\left(\max _{v \in \Gamma_{2^{i} \sqrt{\epsilon}}} \bar{\psi}_{v}^{N} \geq m_{N}+x-2^{i} \sqrt{\epsilon} \mid \Gamma_{2^{i} \sqrt{\epsilon}}\right)\right] .
\end{aligned}
$$

By Fels and Hartung (2019, Proposition 5.1), the second term on the right hand side in (4.15) is bounded from above by

$$
\sum_{i=0}^{\infty} \mathbb{E}\left[\mathbb{P}\left(\max _{v \in V_{N}: v / N \in A} \bar{\psi}_{v}^{N} \geq m_{N}+x-2^{i} \sqrt{\epsilon} \mid \Gamma_{2^{i} \sqrt{\epsilon}}\right)\right] \leq \tilde{c} e^{-c x} \sum_{i=0}^{\infty} \mathbb{E}\left[\frac{\left|\Gamma_{2^{i} \sqrt{\epsilon}}\right|}{\left|\left\{v \in V_{N}: v / N \in A\right\}\right|}\right] e^{c 2^{i} \sqrt{\epsilon}},
$$

where $\tilde{c}>0$ is a finite constant. By assumption (4.13), one has

$$
\mathbb{E}\left[\frac{\left|\Gamma_{2^{i} \sqrt{\epsilon}}\right|}{\left|\left\{v \in V_{N}: v / N \in A\right\}\right|}\right] \leq e^{-4^{i}(C \epsilon)^{-1}} .
$$

Thus, using (4.17), (4.16) is bounded from above by

$$
\tilde{c} e^{-c x} e^{-(C \epsilon)^{-1}}
$$

This concludes the proof of Lemma 4.4.

Lemma 4.5. Let $\left\{\tilde{\psi}_{v}^{N}\right\}_{v \in V_{N}}$ be a centred Gaussian field satisfying

$$
\left|\operatorname{Var} \psi_{v}^{N}-\operatorname{Var} \tilde{\psi}_{v}^{N}\right| \leq \epsilon .
$$

Further, fix some $p \in \mathbb{N}$, and disjoint open, non-empty sets $A_{1}, \ldots, A_{p} \subset[0,1]^{2}$. If

$$
\mathbb{E}\left[\tilde{\psi}_{v}^{N} \tilde{\psi}_{w}^{N}\right] \leq \mathbb{E}\left[\psi_{v}^{N} \psi_{w}^{N}\right]+\epsilon
$$

then

$$
\limsup _{N \rightarrow \infty} \tilde{d}\left(\left(\psi_{N, A_{1}}^{*}-m_{N}, \ldots, \psi_{N, A_{p}}^{*}-m_{N}\right),\left(\tilde{\psi}_{N, A_{1}}^{*}-m_{N}, \ldots, \tilde{\psi}_{N, A_{p}}^{*}-m_{N}\right)\right) \leq l(\epsilon),
$$

and else if,

$$
\mathbb{E}\left[\tilde{\psi}_{v}^{N} \tilde{\psi}_{w}^{N}\right]+\epsilon \geq \mathbb{E}\left[\psi_{v}^{N} \psi_{w}^{N}\right]
$$


then

$$
\limsup _{N \rightarrow \infty} \tilde{d}\left(\left(\tilde{\psi}_{N, A_{1}}^{*}-m_{N}, \ldots, \tilde{\psi}_{N, A_{p}}^{*}-m_{N}\right),\left(\psi_{N, A_{1}}^{*}-m_{N}, \ldots, \psi_{N, A_{p}}^{*}-m_{N}\right)\right) \leq l(\epsilon)
$$

where $l(\epsilon) \rightarrow 0$ as $\epsilon \rightarrow 0$.

Proof: Let $\left\{\psi_{v}^{N}\right\}_{v \in V_{N}},\left\{\tilde{\psi}_{v}^{N}\right\}_{v \in V_{N}}$ satisfy relations (4.19) and (4.20). Let $\Phi,\left\{\Phi_{v}^{N}\right\}_{v \in V_{N}}$ be a collection of independent standard Gaussian random variables, and $\epsilon^{*}(\epsilon)>0$. For $v \in V_{N}$, set

$$
\begin{aligned}
& \psi_{v}^{N, l w, \epsilon^{*}}=\left(1-\frac{\epsilon^{*}}{\log N}\right) \psi_{v}^{N}+\epsilon^{N, \prime} \Phi, \\
& \tilde{\psi}_{v}^{N, u p, \epsilon^{*}}=\left(1-\frac{\epsilon^{*}}{\log N}\right) \tilde{\psi}_{v}^{N}+\epsilon_{v}^{N, \prime \prime} \Phi_{v}^{N},
\end{aligned}
$$

where we can choose, as in the proof of Fels and Hartung (2019, Lemma 5.6), $\epsilon^{*}, \epsilon_{v}^{N, \prime}=\epsilon_{v}^{N, \prime}\left(\epsilon, \epsilon^{*}\right)$ and $\epsilon_{v}^{N, \prime \prime}=\epsilon_{v}^{N, \prime \prime}\left(\epsilon, \epsilon^{*}\right)$ all non-negative and tending to 0 as $\epsilon \rightarrow 0$, such that

$$
\operatorname{Var}\left[\psi_{v}^{N, l w, \epsilon^{*}}\right]=\operatorname{Var}\left[\tilde{\psi}_{v}^{N, u p, \epsilon^{*}}\right]=\operatorname{Var}\left[\psi_{v}^{N}\right]+\epsilon, \quad \forall v \in V_{N}
$$

and

$$
\mathbb{E}\left[\psi_{v}^{N, l w, \epsilon^{*}} \psi_{w}^{N, l w, \epsilon^{*}}\right] \geq \mathbb{E}\left[\tilde{\psi}_{v}^{N, u p, \epsilon^{*}} \tilde{\psi}_{w}^{N, u p, \epsilon^{*}}\right], \quad \forall v, w \in V_{N} .
$$

An application of Theorem 5.2, a version of Slepian's lemma for vectors, gives

$$
\tilde{d}\left(\left(\psi_{N, l w, \epsilon^{*}, A_{1}}^{*}-m_{N}, \ldots, \psi_{N, l w, \epsilon^{*}, A_{p}}^{*}-m_{N}\right),\left(\tilde{\psi}_{N, u p, \epsilon^{*}, A_{1}}^{*}-m_{N}, \ldots, \tilde{\psi}_{N, u p, \epsilon^{*}, A_{1}}^{*}-m_{N}\right)\right)=0 .
$$

By Lemma 4.4, we obtain, for $x_{1}, \ldots, x_{p} \in \mathbb{R}$,

$$
\begin{aligned}
\mathbb{P}\left(\tilde{\psi}_{N, u p, \epsilon^{*}, A_{i}}^{*}-m_{N} \geq x_{i}, 1 \leq i \leq p\right) \leq \mathbb{P} & \left(\psi_{N, A_{i}}^{*}-m_{N} \geq x_{i}-\sqrt{\max _{w \in V_{N}} \epsilon_{w}^{N, \prime \prime}}, 1 \leq i \leq p\right) \\
& \times C e^{-\left(C \max _{w \in V_{N}} \epsilon_{w}^{N, \prime \prime}\right)^{-1}} .
\end{aligned}
$$

Since $\lim _{\epsilon \rightarrow 0} \max _{w \in V_{N}} \epsilon_{w}^{N, \prime \prime}=0$ this implies (4.21). (4.23) can be proved the same way by switching the roles of $\left\{\psi_{v}^{N}\right\}_{v \in V_{N}}$ and $\left\{\tilde{\psi}_{v}^{N}\right\}_{v \in V_{N}}$. We omit further details.

Proposition 4.6. Let $\tilde{\sigma}=\left(\tilde{\sigma}_{1}, \tilde{\sigma}_{2}\right) \in(0, \infty)^{2}, r=\left(r_{1}, r_{2}\right) \in(0, \infty)^{2}$, and $\left\{\psi_{v}^{N, r, \tilde{\sigma}}: v \in V_{N}\right\}$ as well as $\left\{\psi_{v}^{N, \tilde{\sigma}, *}: v \in V_{N}\right\}$ be two Gaussian fields given by

$$
\psi_{v}^{N, r, \tilde{\sigma}}=\psi_{v}^{N}+\tilde{\sigma}_{1} g_{B_{v, r_{1}}}+\tilde{\sigma}_{2} g_{B_{v, N / r_{2}}}, \quad \text { for } v \in V_{n}
$$

and

$$
\psi_{v}^{N, \tilde{\sigma}, *}=\psi_{v}^{N}+\sqrt{\frac{\|\tilde{\sigma}\|_{2}^{2}}{\log (N)}} \tilde{\psi}_{v}^{N}, \quad \text { for } v \in V_{N}
$$

where $\left\{\psi_{v}^{N}\right\}_{v \in V_{N}},\left\{\tilde{\psi}_{v}^{N}\right\}_{v \in V_{N}}$ are two independent scale-inhomogeneous DGFFs, satisfying Assumption 1.3, and where $\left\{g_{B}\right\}_{B \in \mathcal{B}}$ is a collection of independent standard Gaussians. For a set $A \subset[0,1]^{2}$,

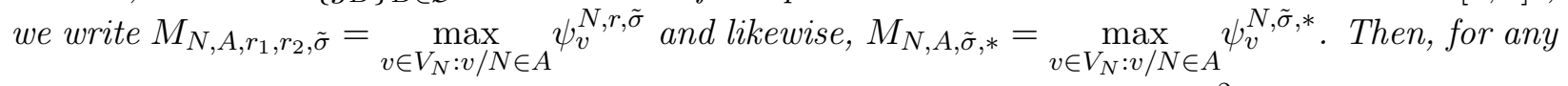
$p \in \mathbb{N}$, and any collection of disjoint, open and non-empty $A_{1}, \ldots, A_{p} \subset[0,1]^{2}$,

$$
\begin{aligned}
\limsup _{N \rightarrow \infty} d\left(\left(M_{N, A_{1}, r_{1}, r_{2}, \tilde{\sigma}}-m_{N}, \ldots, M_{N, A_{p}, r_{1}, r_{2}, \tilde{\sigma}}-m_{N}\right),\right. \\
\left.\left(M_{N, A_{1}, \tilde{\sigma}, *}-m_{N}, \ldots, M_{N, A_{p}, \tilde{\sigma} *}-m_{N}\right)\right)=0,
\end{aligned}
$$

as $r_{1}, r_{2} \rightarrow \infty$ 
Proof: The proof is a straightforward adaptation of the proof of Fels and Hartung (2019, Proposition B.2). Decompose $V_{N}$ into boxes $B$ of side length $N / r_{2}$ and call their collection $\mathcal{B}$. Further, for $\delta \in(0,1)$ and $B \in \mathcal{B}$, let $B_{\delta}$ be the box with the identical centre as $B$, and reduced side length $(1-\delta) N / r_{2}$. Then, we set $V_{N, \delta}=\cup_{B \in \mathcal{B}} B_{\delta}$. The corresponding maxima over are called $M_{N, A, r_{1}, r_{2}, \tilde{\sigma}, \delta}=\max _{v \in V_{N, \delta}: v / N \in A} \psi_{v}^{N, r, \tilde{\sigma}}$ and $M_{N, A, \tilde{\sigma}, *}=\max _{v \in V_{N, \delta}: v / N \in A} \psi_{v}^{N, \tilde{\sigma}, *}$. Fels and Hartung (2019, Proposition 5.1) shows that it suffices to consider the maxima on the slightly smaller sets, i.e. one has

$$
\begin{array}{r}
\lim _{\delta \rightarrow 0} \lim _{N \rightarrow \infty} \mathbb{P}\left(M_{N, A_{1}, r_{1}, r_{2}, \tilde{\sigma}, \delta} \neq M_{N, A_{1}, r_{1}, r_{2}, \tilde{\sigma}}, \ldots, M_{N, A_{p}, r_{1}, r_{2}, \tilde{\sigma}, \delta} \neq M_{N, A_{p}, r_{1}, r_{2}, \tilde{\sigma}}\right) \\
=\lim _{\delta \rightarrow 0} \lim _{N \rightarrow \infty} \mathbb{P}\left(M_{N, A_{1}, \tilde{\sigma}, *, \delta} \neq M_{N, A_{1}, \tilde{\sigma}, *}, \ldots, M_{N, A_{p}, \tilde{\sigma}, *, \delta} \neq M_{N, A_{p}, \tilde{\sigma}, *}\right)=0 .
\end{array}
$$

Next, we claim that the maximum is essentially determined by the maximum of the unperturbed scale-inhomogeneous DGFF, $\left\{\psi_{v}^{N}\right\}_{v \in V_{N}}$. For $B \in \mathcal{B}$, let $z_{B}$ be the unique element, such that

$$
\psi_{z_{B}}^{N}=\max _{v \in B_{\delta}} \psi_{v}^{N}
$$

The claim is that

$$
\begin{aligned}
\lim _{r_{1}, r_{2} \rightarrow \infty} \lim _{N \rightarrow \infty} \mathbb{P} & \left(\left|M_{N, A_{i}, r_{1}, r_{2}, \tilde{\sigma}, \delta}-\max _{B \in \mathcal{B}, B \subset N A_{i}} \psi_{z_{B}}^{N, r, \tilde{\sigma}}\right| \geq \frac{1}{\log n}: 1 \leq i \leq p\right) \\
& =\limsup _{N \rightarrow \infty} \mathbb{P}\left(\left|M_{N, A_{i}, \tilde{\sigma}, *, \delta}-\max _{B \in \mathcal{B}, B \subset N A_{i}} \psi_{z_{B}}^{N, \tilde{\sigma}, *}\right| \geq \frac{1}{\log n}: 1 \leq i \leq p\right)=0,
\end{aligned}
$$

with $N A_{i}=\left\{v \in \mathbb{Z}^{2}: v / N \in A_{i}\right\}$. In the following, we show that none of the events in the probabilities in (4.35) can occur. It suffices to show that none of the following events can happen. For $i \in\{1, \ldots, p\}$, let

$$
\begin{aligned}
E_{1}^{(i)}= & \left\{M_{N, A_{i}, r_{1}, r_{2}, \tilde{\sigma}, \delta} \notin\left(m_{N}-C, m_{N}+C\right)\right\} \cup\left\{M_{N, A_{i}, \tilde{\sigma}, *, \delta} \notin\left(m_{N}-C, m_{N}+C\right)\right\} \\
E_{2}^{(i)}= & \left\{\exists u, v \in V_{N}: u, v / N \in A_{i},\|u-v\| \in(r, N / r) \text { and } \min \left(\psi_{u}^{N}, \psi_{v}^{N}\right)>m_{N}-c \log n\right\} \\
E_{3}^{(i)}= & \tilde{E}_{3}^{(i)} \cup \bar{E}_{3}^{(i)}, \\
& \text { where } \tilde{E}_{3}^{(i)}=\left\{\omega: \exists v \in V_{N}, v / N \in A_{i}: \psi_{v}^{N, r, \tilde{\sigma}}=M_{N, A_{i}, r_{1}, r_{2}, \tilde{\sigma}, \delta}, \psi_{v}^{N} \leq m_{N}-c \log n\right\}, \\
\bar{E}_{3}^{(i)}= & \left\{\omega: \exists v \in V_{N}, v / N \in A_{i}: \psi_{v}^{N, \tilde{\sigma}, *}=M_{N, A_{i}, \tilde{\sigma}, *, \delta}, \psi_{v}^{N} \leq m_{N}-c \log n\right\} \\
E_{4}^{(i)}= & \left\{\exists v \in B \in \mathcal{B} \subset N A_{i}: \psi_{v}^{N} \geq m_{N}-c \log n \text { and } \sqrt{\frac{\|\tilde{\sigma}\|_{2}^{2}}{\log N}} \tilde{\psi}_{v}^{N}-\sqrt{\left.\frac{\|\tilde{\sigma}\|_{2}^{2}}{\log N} \tilde{\psi}_{z_{B}}^{N} \geq 1 / \log n\right\} .}\right.
\end{aligned}
$$

The events, $E_{2}^{(i)}, E_{3}^{(i)}, E_{4}^{(i)}$, we are considering here, are subsets of the events, $E_{2}, E_{3}$ and $E_{4}$, which appear in the proof of Fels and Hartung (2019, Proposition B.2). In the proof of Fels and Hartung (2019, Proposition B.2), we showed that their probabilities tend to zero, and thus, we may conclude the same for the events, $E_{2}^{(i)}, E_{3}^{(i)}, E_{4}^{(i)}$. So, we are left with bounding the events $E_{1}^{(i)}$. First note that it suffices to consider the scale-inhomogeneous DGFF, as the other terms are centred Gaussians with uniformly bounded variance. Since maximizing over a subset, we have, for any $i \in\{1, \ldots, p\}$,

$$
\mathbb{P}\left(\max _{v \in V_{N}: v / N \in A_{i}} \psi_{v}^{N}>m_{N}+C\right) \leq \mathbb{P}\left(\max _{v \in V_{N}} \psi_{v}^{N}>m_{N}+C\right)
$$


By tightness of the centred maximum Fels and Hartung $(2019,(2.2)),(4.40)$ tends to 0 as $C \rightarrow \infty$, uniformly in $N$. Hence to show (4.35), it suffices to prove, for any $i \in\{1, \ldots, p\}$,

$$
\lim _{C \rightarrow \infty} \lim _{N \rightarrow \infty} \mathbb{P}\left(\max _{v \in V_{N}: v / N \in A_{i}} \psi_{v}^{N} \leq m_{N}-C\right)=0
$$

Assume otherwise, then there is a subsequence $\left\{N_{k}\right\}_{k \in \mathbb{N}}$, a sequence $C_{N} \rightarrow \infty$ as $N \rightarrow \infty$ and a constant $\epsilon>0$, such that, for any $k \in \mathbb{N}$,

$$
\mathbb{P}\left(\max _{v \in V_{N_{k}}: v / N_{k} \in A_{i}} \psi_{v}^{N_{k}} \leq m_{N_{k}}-C_{N_{k}}\right) \geq \epsilon .
$$

We can further assume that $A_{i} \subset[0,1]^{2}$ is a box, otherwise pick the largest box that fits into $A_{i}$. We can decompose $[0,1]^{2}$ into disjoint translations of $A_{i}^{(j)}$, that we possible need to cut with $[0,1]^{2}$. For each $A_{i}^{(j)} N$ we consider an independent copy of $\left\{\psi_{v}^{N}\right\}_{v \in V_{N}}$, called $\left\{\psi_{v}^{N, j}\right\}_{v \in V_{N}}$. By translation invariance, for each of these (4.42) holds. By Gaussian comparison, independence and (4.42), we have

$$
\mathbb{P}\left(\max _{v \in V_{N_{k}}} \psi_{v}^{N_{k}} \leq m_{N_{k}}-C_{N_{k}}\right) \geq \mathbb{P}\left(\max _{j} \max _{v / N_{k} \in A_{i}^{(j)}} \psi_{v}^{N_{k}, j} \leq m_{N_{k}}-C_{N_{k}}\right)>0 .
$$

By tightness of $\left\{\max _{v \in V_{N}} \psi_{v}^{N}-m_{N}\right\}_{N \in \mathbb{N}}$, the left-hand side of (4.43) tends to zero, which is a contradiction. Thus, this yields (4.41), which concludes the proof of Proposition 4.6.

Lemma 4.5 and Proposition 4.6 allow us to prove Lemma 4.3.

Proof of Lemma 4.3: Define for $v \in V_{N}, \bar{\psi}_{v}^{N, \tilde{\sigma}}=\left(1+\frac{\|\tilde{\sigma}\|^{2}}{\log (N)}\right) \psi_{v}^{N}$, and for $A \subset[0,1]^{2}$ open and non-empty, $\bar{M}_{N, A, \tilde{\sigma}}=\max _{v \in V_{N}: v / N \in A} \bar{\psi}_{v}^{N, \tilde{\sigma}}$ and set $M_{N, A}=\max _{v \in V_{N}: v / N \in A} \psi_{v}^{N}$. (4.40) together with tightness of the centred maximum Fels and Hartung (2019, (2.2)) and (4.41) implies,

$$
\mathbb{E}\left[\bar{M}_{N, A_{i}, \tilde{\sigma}}\right]=\mathbb{E}\left[M_{N, A_{i}}\right]+2\|\tilde{\sigma}\|_{2}^{2}+o(1)
$$

and

$$
\lim _{N \rightarrow \infty} d\left(M_{N, A_{i}}-\mathbb{E}\left[M_{N, A_{i}}\right], \bar{M}_{N, A_{i}, \tilde{\sigma}}-\mathbb{E}\left[\bar{M}_{N, A_{i}, \tilde{\sigma}}\right]\right)=0
$$

Next, we consider the field, $\left\{\psi_{v}^{N, \tilde{\sigma}, *}\right\}_{v \in V_{N}}$, defined in (4.31). For $i \in\{1, \ldots, p\}$, set

$$
M_{N, A_{i}, \tilde{\sigma}, *}=\max _{v \in V_{N}: v / N \in A_{i}} \psi_{v}^{N, \tilde{\sigma}, *} .
$$

In distribution, $\left\{\psi_{v}^{N, \tilde{\sigma}, *}\right\}_{v \in V_{N}}$ can be written as a sum of $\left\{\bar{\psi}_{v}^{N, \tilde{\sigma}}\right\}_{v \in V_{N}}$ and an independent centred Gaussian field with variances of order $O\left((1 / \log N)^{3}\right)$. Thus, by Gaussian comparison,

$$
\mathbb{E}\left[\bar{M}_{N, A_{i}, \tilde{\sigma}}\right]=\mathbb{E}\left[M_{N, A_{i}, \tilde{\sigma}, *}\right]+o(1)
$$

and

$$
\lim _{N \rightarrow \infty} d\left(\left(\bar{M}_{N, A_{i}, \tilde{\sigma}}-\mathbb{E}\left[\bar{M}_{\left.N, A_{i}, \tilde{\sigma}\right]}\right]\right)_{1 \leq i \leq p},\left(\bar{M}_{N, A_{i}, \tilde{\sigma}, *}-\mathbb{E}\left[\bar{M}_{N, A_{i}, \tilde{\sigma}, *}\right]\right)_{1 \leq i \leq p}\right)=0 .
$$

Combining (4.48) with Proposition 4.6 and applying the triangle inequality, one concludes the proof of Lemma 4.3. 
4.3. Coupling to independent random variables. Recall $\underline{A}=\left(A_{1}, \ldots, A_{p}\right)$ is a collection of disjoint open, non-empty, simply-connected subsets of $[0,1]^{2}$, for some fixed $p \in \mathbb{N}$. Further, we have tiled $V_{N}$ with boxes $B_{N / K L, i}$ of side length $N / K L$. Instead of considering the maximum over the sets $\left\{v \in V_{N}: v / N \in A_{i}\right\}$, we want to work with the $B_{N / K L}$-boxes. Thus, for any $i \in\{1, \ldots, p\}$, let $T_{i}^{(K L)} \subset\left\{1, \ldots,(K L)^{2}\right\}$ denote the maximal index set, such that $j \in T_{i}^{(K L)}$ implies $B_{N / K L, j} \subset N A_{i}$, i.e.

$$
\cup_{j \in T_{i}^{(K L)}} B_{N / K L, j} \subset N A_{i},
$$

with $N A_{i}=\left\{v \in \mathbb{Z}^{2}: v / N \in A_{i}\right\}$. Further, it is immediate to see that for all $1 \leq i \leq p$

$$
\frac{\left|N A_{i} \backslash \cup_{j \in T_{i}^{(K L)}} B_{N / K L, j}\right|}{\left|N A_{i}\right|} \rightarrow 0,
$$

as we let $N, K, L$ tend to infinity in this order and where we write $\left|N A_{i}\right|=\left|N A_{i} \cap \mathbb{Z}^{2}\right|$. In particular,

$$
\begin{aligned}
& \mathbb{P}\left(\max _{v \in \cup_{i=1}^{p}\left\{A_{i} \backslash\left\{\cup_{j \in T_{i}^{(K L)}} B_{N / K L, j}\right\}\right\}} \psi_{v}^{N} \geq m_{N}+z\right) \\
& \leq \sum_{i=1}^{p}\left|N A_{i} \backslash \cup_{j \in T_{i}^{(K L)}} B_{N / K L, j}\right| \sup _{v \in V_{N}} \mathbb{P}\left(\psi_{v}^{N} \geq m_{N}+z\right) \\
& \leq C \sum_{i=1}^{p} \frac{\left|N A_{i} \backslash \cup_{j \in T_{i}^{(K L)}} B_{N / K L, j}\right|}{N^{2}} e^{-2 z},
\end{aligned}
$$

which, by (4.50), converges to zero as $N \rightarrow \infty$. Next, we construct random variables that do not depend on $N$ and that we couple to the local maxima of $\left\{S_{v}^{N}\right\}_{v \in V_{N}}$ on $\cup_{j \in T_{1}^{(K L)}} B_{N / K L, j}, \ldots$, $\cup_{j \in T_{p}^{(K L)}} B_{N / K L, j}$. We set, $A_{i}^{\prime}:=\cup_{j \in T_{i}^{(K L)}} B_{N / K L, j}$, and $S_{v}^{N, f}:=S_{v}^{N}-S^{N, c} v$, for $v \in V_{N}$. Set, for $k \leq n$ and $t \in[k, n]$,

$$
m_{N}(k, t):=2 \log N\left(\mathcal{I}_{\sigma^{2}}\left(\frac{t}{n}\right)-\mathcal{I}_{\sigma^{2}}\left(\frac{k}{n}\right)\right)-\frac{\left(t \wedge\left(n-\left(k^{\prime}+l^{\prime}\right)\right)\right) \log n}{4\left(n-\left(k^{\prime}+l^{\prime}\right)\right)} .
$$

Fix $\gamma \in(1 / 2,1)$ and let $\left\{\varrho_{K L, i}: 1 \leq i \leq(K L)^{2}\right\}$ be a collection of independent Bernoulli random variables with

$$
\mathbb{P}\left(\varrho_{K L, i}=1\right)=\beta e^{2(k+l)^{\gamma}} e^{2(k+l)\left(\sigma^{2}(0)-1\right)},
$$

where, by using Fels and Hartung (2019, Proposition 5.8) and Fels and Hartung (2019, (5.70)), the constant $\beta>0$ is the limit of constants $\beta_{K^{\prime}, L^{\prime}}^{*}$, as $K^{\prime} \rightarrow \infty$ and $L^{\prime} \rightarrow \infty$, where the latter are such that by Fels and Hartung (2019, Proposition 5.8) they satisfy

$$
\begin{gathered}
\lim _{z \rightarrow \infty} \limsup _{\left(L^{\prime}, K^{\prime}, N\right) \Rightarrow \infty} \mid e^{2 \log (2)(k+l)\left(1-\sigma^{2}(0)\right)} e^{-2(k+l)^{\gamma}} e^{2 z} \mathbb{P}\left(\max _{v \in B_{N / K L, i}} S_{v}^{N, f} \geq m_{N}(k+l, n)-(k+l)^{\gamma}+z\right) \\
-\beta_{K^{\prime}, L^{\prime}}^{*} \mid=0 .
\end{gathered}
$$

Moreover, the collection, $\left\{\beta_{K^{\prime}, L^{\prime}}^{*}\right\}_{K^{\prime}, L^{\prime} \geq 0}$, and thus, $\beta$, depends on the variance only through $\sigma(1)$. Each of the Bernoulli random variables indicates, for $i=1, \ldots,(K L)^{2}$, that the maximum of fine field, $\left\{S_{v}^{N, f}\right\}_{v \in V_{N}}$, in a box $B_{N / K L, i}$ exceeds the extremal threshold, $m_{N}(k+l, n)-(k+l)^{\gamma}$. In 
particular, the right-hand side of (4.53) is less than one, since by Assumption 1.3, $\sigma(0)<1$, and due to the fact that $\gamma<1$, while we may assume that $k$ is large. In addition, we specify an independent family of exponential random variables, $\left\{Y_{K L, i}: 1 \leq i \leq(K L)^{2}\right\}$,

$$
\mathbb{P}\left(Y_{K L, i} \geq x\right)=e^{-2 x} e^{2(k+l)^{\gamma}}, \quad \text { for } x \geq-(k+l)^{\gamma} .
$$

These specify the exact height, $x$, by which the maximum of the fine field exceeds the extremal threshold, $m_{N}(k+l, n)-(k+l)^{\gamma}$, in each box, $B_{N / K L, i}$, for $i=1, \ldots,(K L)^{2}$. Also, let $\left\{Z_{K L, i}\right\}_{1 \leq i \leq(K L)^{2}}$ be a centered Gaussian field with covariance kernel $\Sigma^{c}(i, j)=\sigma^{2}(0) \mathbb{E}\left[\phi_{w_{i}}^{K L} \phi_{w_{j}}^{K L}\right]$, for $i, j=1, \ldots,(K L)^{2}$, and with $w_{i}$ the vertices of $V_{K L}$ as in (4.1). It captures initial contributions from the largest scales. For each $i \in\{1, \ldots, p\}$, set

$$
G_{L, K, L^{\prime}, K^{\prime}}^{(i)}:=\max _{\substack{j \in T_{i}^{(K L)} \\ \varrho_{K L, j}=1}}\left(Y_{K L, j}+2 \log (K L)\left(1-\sigma^{2}(0)\right)\right)+\left(Z_{K L, j}-2 \log (K L)\right) .
$$

We collect these in the vector

$$
G_{\underline{A}, L, K, L^{\prime}, K^{\prime}}^{*}:=\left(G_{L, K, L^{\prime}, K^{\prime}}^{(1)}, \ldots, G_{L, K, L^{\prime}, K^{\prime}}^{(p)}\right) .
$$

We denote the law of the random vector defined in (4.57) by $\bar{\mu}_{L, K, L^{\prime}, K^{\prime}, \underline{A}}$, which does not depend on $N$. Next, we show that $\bar{\mu}_{L, K, L^{\prime}, K^{\prime}, \underline{A}}$ converges to the same limit as $\mu_{N, \underline{A}}$, the law of

$$
\left(\max _{v \in A_{1}^{\prime}} S_{v}^{N}-m_{N}, \ldots, \max _{v \in A_{p}^{\prime}} S_{v}^{N}-m_{N}\right)
$$

The following theoroem establishes that $\bar{\mu}_{L, K, L^{\prime}, K^{\prime}, \underline{A}}$ is a good approximation for $\mu_{N, \underline{A}}$. The key idea is a localization of extremely large vertices as in Proposition 3.3. To have an extreme local maximum, the fine field has to be much larger than usual. In fact, we need that on the leading order the fine field is roughly at height $2 \log N\left(1-\mathcal{I}_{\sigma^{2}}\left(\frac{k+l}{n}\right)\right)$ which, due to Assumption 1.3, linearily exceeds the leading order of its expected maximum on a box of side length $N / K L$, which is $2 \log N \sqrt{\left(1-\mathcal{I}_{\sigma^{2}}\left(\frac{k+l}{n}\right)\right)}$. Considering the right-tail asymptotics for the fine field in (4.54), this is well captured by the collection of random variables $\left(\varrho_{K L, i}, Y_{K L, i}\right)_{i=1, \ldots,(K L)^{2}}$ by construction. The exact height of an extreme local maximum is then simply given by the sum of the value of the coarse field and the height of the extremely large fine field.

Theorem 4.7. It holds that

$$
\limsup _{\left(L, K, L^{\prime}, K^{\prime}, N\right) \Rightarrow \infty} d\left(\mu_{N, \underline{A}}, \bar{\mu}_{L, K, L^{\prime}, K^{\prime}, \underline{A}}\right)=0 .
$$

In particular, there exists $\mu_{\infty, \underline{A}}$ such that $\lim _{\left(L, K, L^{\prime}, K^{\prime}, N\right) \Rightarrow \infty} d\left(\mu_{N, \underline{A}}, \mu_{\infty, \underline{A}}\right)=0$.

Proof: We follow the proof of Fels and Hartung (2019, Theorem 5.9) that deals with the global maximum. First, note that by the exact same truncated second moment computations as in Fels (2019), just taking the sum over reduced sets, we have tightness of the centred maxima of the scaleinhomogeneous DGFF on subsets $N A_{i}$, for $i=1, \ldots, p$, provided $\operatorname{vol}\left(A_{i}\right)>0$. By Lemma 4.2, the same holds for centred maxima on subsets $N A_{i}$, for $i=1, \ldots, p$, of the 3-field approximation. In particular, joint convergence holds on a subsequence. Denote by $\tau_{i}^{\prime}=\arg \max _{v \in B_{N / K L, i}} S_{v}^{N}$, the a.s. unique point where the local maximum is achieved. By Fels and Hartung (2019, (5.50)), we have, for $1 \leq i \leq p$,

$$
\limsup _{\left(L, K, L^{\prime}, K^{\prime}, N\right) \Rightarrow \infty} \mathbb{P}\left(S_{\tau_{i}^{\prime}}^{N, f} \geq m_{N}(k+l, n)-(k+l)^{\gamma}\right)=1
$$


Moreover, we know that the fine field values cannot be too large, i.e. let

$$
\begin{aligned}
& \mathcal{E}=\cup_{1 \leq i \leq(K L)^{2}}\left\{\max _{v \in B_{N / K L, i}} S_{v}^{N, f} \geq m_{N}(k+l, n)+K L+(k+l)^{\gamma}\right\}, \text { and } \\
& \mathcal{E}^{\prime}=\cup_{1 \leq i \leq(K L)^{2}}\left\{Y_{K L, i} \geq K L+(k+l)^{\gamma}\right\} .
\end{aligned}
$$

By Fels and Hartung (2019, (5.51)) respectively Fels and Hartung (2019, (5.53)), we deduce

$$
\limsup _{\left(L, K, L^{\prime}, K^{\prime}, N\right) \Rightarrow \infty} \mathbb{P}(\mathcal{E})=0 \text { and } \limsup _{\left(L, K, L^{\prime}, K^{\prime}, N\right) \Rightarrow \infty} \mathbb{P}\left(\mathcal{E}^{\prime}\right)=0 .
$$

This allows to couple the centred fine field, $\tilde{M}_{N, i}^{f}=\max _{v \in B_{N / K L, i}} S_{i}^{N, f}-m_{N}((k+l), n)$, to the approximating process $G_{L, K, L^{\prime}, K^{\prime}}^{(i)}$, defined in (4.56). By Fels and Hartung (2019, Proposition 5.8), there are $\epsilon_{N, K L, K^{\prime} L^{\prime}}^{*}>0$ with

$$
\limsup _{\left(L, K, L^{\prime}, K^{\prime}, N\right) \Rightarrow \infty} \epsilon_{N, K L, K^{\prime} L^{\prime}}^{*}=0,
$$

such that, for some $|\hat{\epsilon}| \leq \epsilon_{N, K L, K^{\prime} L^{\prime}}^{*} / 4$,

$$
\mathbb{P}\left(-(k+l)^{\gamma}+\stackrel{\bullet}{\epsilon} \leq \tilde{M}_{N, i}^{f} \leq K L+(k+l)^{\gamma}\right)=\mathbb{P}\left(\varrho_{K L, i}=1, Y_{K L, i} \leq K L+(k+l)^{\gamma}\right),
$$

and such that for all $t$ with $-(k+l)^{\gamma}-1 \leq t \leq K L+(k+l)^{\gamma}$,

$$
\begin{aligned}
\mathbb{P}\left(\varrho_{K L, i}=1, Y_{K L, i} \leq t-\epsilon_{N, K L, K^{\prime} L^{\prime}}^{*}\right) & \leq \mathbb{P}\left(-(k+l)^{\gamma}+\stackrel{\diamond}{\epsilon} \leq \tilde{M}_{N, i}^{f} \leq t\right) \\
& \leq \mathbb{P}\left(\varrho_{K L, i}=1, Y_{K L, i} \leq t+\epsilon_{N, K L, K^{\prime} L^{\prime}}^{*} / 2\right) .
\end{aligned}
$$

Thus, there is a coupling between $\left\{\tilde{M}_{N, i}^{f}: 1 \leq i \leq(K L)^{2}\right\}$ and $\left\{\left(\varrho_{K L, i}, Y_{K L, i}\right): 1 \leq i \leq(K L)^{2}\right\}$ such that on the event $\left(\mathcal{E} \cup \mathcal{E}^{\prime}\right)^{c}$ :

$$
\begin{aligned}
& \varrho_{K L, i}=1,\left|Y_{K L, i}-\tilde{M}_{N, i}^{f}\right| \leq \epsilon_{N, K L, K^{\prime} L^{\prime}}^{*}, \quad \text { if } \tilde{M}_{N, i}^{f} \geq \epsilon_{N, K L, K^{\prime} L^{\prime}}^{*} \\
& \left|Y_{K L, i}-\tilde{M}_{N, i}^{f}\right| \leq \epsilon_{N, K L, K^{\prime} L^{\prime}}^{*}, \quad \text { if } \varrho_{K L, i}=1 .
\end{aligned}
$$

A short argument for the existence of such couplings and which is taken from the proof of Fels and Hartung (2019, Theorem 5.9) is as follows: In the event $\mathcal{E}^{c} \cap \mathcal{E}^{\prime, c},(4.64)$ becomes

$$
\mathbb{P}\left(-(k+l)^{\gamma}+\epsilon^{\diamond} \leq M_{n, i}^{f}\right)=\mathbb{P}\left(\varrho_{K L, i}=1\right) .
$$

By (4.65) and since the random variables have distributions that are absolutely continuous with respect to the Lebesgue measure, there is an increasing function, $g: \mathbb{R} \rightarrow \mathbb{R}$, with $g(t) \in\left[t-\epsilon^{*} / 2, t+\right.$ $\left.\epsilon^{*} / 2\right]$, for $-\bar{k}^{\gamma}-1 \leq t \leq K L+\bar{k}^{\gamma}$, and such that

$$
\mathbb{P}\left(\varrho_{K L, i}=1, Y_{K L, i} \leq g(t)\right)=\mathbb{P}\left(-(k+l)^{\gamma}+\epsilon^{\diamond} \leq M_{n, i}^{f} \leq t\right) .
$$

Let $-(k+l)^{\gamma}-1=t_{0}<\ldots<t_{D}=K L+(k+l)^{\gamma}$ be an arbitrary partition. Define sets

$$
\begin{aligned}
& A_{j}:=\left\{\omega: \varrho_{K L, i}(\omega)=1, Y_{K L, i}(\omega) \in\left[g\left(t_{j}\right), g\left(t_{j+1}\right)\right)\right\}, \\
& B_{j}:=\left\{\omega: \epsilon^{\diamond} \leq M_{n, i}^{f}(\omega) \in\left[t_{j}, t_{j+1}\right)\right\} .
\end{aligned}
$$

In particular, for any $0 \leq j<D, \mathbb{P}\left(A_{j}\right)=\mathbb{P}\left(B_{j}\right)$. Define random variables $\left(\varrho_{K L, i}^{\prime}, Y_{K L, i}^{\prime}\right)$, i.e for $\omega \in$ $B_{j} \cap\left(\mathcal{E} \cup \mathcal{E}^{\prime}\right)^{c}$, set $Y_{K L, i}^{\prime}(\omega)=g\left(M_{n, i}^{f}(\omega)\right)$ and such that, for all $\omega \in\left(\mathcal{E} \cup \mathcal{E}^{\prime}\right)^{c} \cap\left(\cup_{j} B_{j}\right), \varrho_{K L, i}^{\prime}(\omega)=$ 1. For $\omega \in \mathcal{E} \cup \mathcal{E}^{\prime}$, set $\varrho_{K L, i}^{\prime}(\omega)=\varrho_{K L, i}(\omega)$ and $Y_{K L, i}^{\prime}(\omega)=Y_{K L, i}(\omega)$. Then $\left(\varrho_{K L, i}^{\prime}, Y_{K L, i}^{\prime}\right) \stackrel{d}{=}$ $\left(\varrho_{K L, i}, Y_{K L, i}\right)$, and $\left(\varrho_{K L, i}^{\prime}, Y_{K L, i}^{\prime}\right)$ additionally satisfies both (4.66) and (4.67). As $\left\{Z_{K L, i}\right\}_{1 \leq i \leq(K L)^{2}}$ 
and $\left\{S_{v}^{N, c}\right\}_{v \in V_{N}}$ have the same law, one can couple such that $S_{v}^{N, c}=Z_{K L, i}$, for $v \in B_{N / K L, i}$ and $1 \leq i \leq(K L)^{2}$. Using Fels and Hartung (2019, (5.63)), we deduce

$$
\limsup _{\left(L, K, L^{\prime}, K^{\prime}, N\right) \Rightarrow \infty} \mathbb{P}\left(\varrho_{K L, \tilde{\tau}_{i}}=1\right)=1
$$

and thereby exclude that the local maximum is achieved in a box $T_{j}^{(K L)}$ when at the same time $\varrho_{K L, j}=0$. Thus, there are couplings, such that outside an event of vanishing probability as $\left(L, K, L^{\prime}, K^{\prime}, N\right) \Rightarrow \infty$, we have

$$
\left(\left(\max _{v \in A_{1}^{\prime}} S_{v}^{N}-m_{N}\right)-G_{L, K, L^{\prime}, K^{\prime}}^{(1)} \ldots,\left(\max _{v \in A_{p}^{\prime}} S_{v}^{N}-m_{N}\right)-G_{L, K, L^{\prime}, K^{\prime}}^{(p)}\right)_{\infty} \leq 2 \epsilon_{N, K L, K^{\prime} L^{\prime}}^{*}
$$

which, together with convergence of $\left(\max _{v \in A_{i}^{\prime}} S_{v}^{N}-m_{N}\right)_{i=1, \ldots, p}$ on a subsequence, proves Theorem 4.7.

Next, we prove Theorem 2.5.

Proof of Theorem 2.5: By Lemma 4.2, (4.51) and Theorem 4.7, we can reduce the proof to proving convergence of the laws $\bar{\mu}_{L, K, L^{\prime}, K^{\prime}, \underline{A}}$. Recall that $\left\{Z_{K L, i}\right\}_{i=1, \ldots, K L}$ is a centred Gaussian field with covariances given by $\mathbb{E}\left[Z_{K L, i} Z_{K L, j}\right]=\sigma^{2}(0) \mathbb{E}\left[\phi_{w_{i}}^{K L} \phi_{w_{j}}^{K L}\right]$, for $i, j=1, \ldots,(K L)^{2}$ and with $w_{i}, w_{j}$ being the vertices of $V_{K L}$ as defined at the beginning of Subsection 4.1. In the following, we construct random variables $\left\{D_{K L}\left(A_{i}\right): 1 \leq i \leq p\right\}_{K, L \geq 0}$ that are measurable with respect to $\mathcal{F}^{C}:=\sigma\left(Z_{K L, i}\right)_{i=1}^{(K L)^{2}}$, so that for any $x_{1}, \ldots, x_{p} \in \mathbb{R}$, the following limit exists

$$
\lim _{\left(L, K, L^{\prime}, K^{\prime}\right) \Rightarrow \infty} \frac{\bar{\mu}_{L, K, L^{\prime}, K^{\prime}, \underline{A}}\left(\left(-\infty, x_{1}\right], \ldots,\left(-\infty, x_{p}\right]\right)}{\mathbb{E}\left[\exp \left(-\beta \sum_{i=1}^{p} D_{K L}\left(A_{i}\right) e^{\left.-2 x_{i}\right)}\right]\right.},
$$

and is equal to one. Regarding (4.72), assume $\varrho_{K L, \tilde{\tau}_{i}}=1$, for $1 \leq i \leq p$. Recalling (4.56), that is the definition of $G_{L, K, L^{\prime}, K^{\prime}}^{(i)}$, for $i=1, \ldots, p$, and conditioning on $\mathcal{F}^{c}$, we have, for any $x_{1}, \ldots, x_{p} \in \mathbb{R}$,

$$
\begin{aligned}
& \bar{\mu}_{L, K, L^{\prime}, K^{\prime}}\left(\left(-\infty, x_{1}\right], \ldots,\left(-\infty, x_{p}\right]\right)=\mathbb{P}\left(G_{L, K, L^{\prime}, K^{\prime}}^{(i)} \leq x_{i}: i=1, \ldots, p\right) \\
& =\mathbb{E}\left[\prod_{i=1}^{p}\left(1-\mathbb{P}\left(\varrho_{K L, j}\left(Y_{K L, j}+2 \log (K L)\left(\sigma_{1}^{2}-1\right)\right)>x_{i}+2 \log (K L)-Z_{K L, j} \mid \mathcal{F}^{c}\right)\right)^{\mid T^{(K L)_{i} \mid}}\right] .
\end{aligned}
$$

A union bound on $\mathcal{D}^{c}=\left\{\min _{1 \leq i \leq(K L)^{2}} 2 \log (K L)-Z_{K L, i} \geq 0\right\}^{c}$, shows that

$$
\limsup _{(L, K) \Longrightarrow \infty} \mathbb{P}(\mathcal{D})=1 .
$$

Thus, on the event $\mathcal{D}$, and by (4.53), (4.55) and (4.79), one deduces

$$
\mathbb{P}\left(\varrho_{K L, j} Y_{K L, j} \geq 2 \log (K L) \sigma^{2}(0)-Z_{K L, j}+x_{i} \mid \mathcal{F}^{c}\right)=\beta e^{-2\left(\left(1+\sigma^{2}(0)\right) \log (K L)-Z_{K L, j}+x_{i}\right)}
$$

In particular, note that (4.77) tends to zero as $K L \rightarrow \infty$. Using $e^{-\frac{x}{1-x}} \leq 1-x \leq e^{-x}$, for $x<1$, and inserting for $x$ the probability in (4.77) with $K, L$ large, implies that there is non-negative sequence 
$\left\{\epsilon_{K, L}\right\}_{K, L \geq 0}$, with $\limsup \epsilon_{K, L}=0$, such that

$$
\begin{aligned}
(L, K) & \Rightarrow \infty \\
& \exp \left(-\left(1+\epsilon_{K, L}\right) \beta e^{-2\left(\left(1+\sigma^{2}(0)\right) \log (K L)-Z_{K L, j}+x_{i}\right)}\right) \\
& \leq \mathbb{P}\left(\varrho_{K L, j} Y_{K L, j} \leq 2 \log (K L) \sigma^{2}(0)-Z_{K L, j}+x_{i} \mid \mathcal{F}^{c}\right) \\
& \leq \exp \left(-\left(1-\epsilon_{K, L}\right) \beta e^{-2\left(\left(1+\sigma^{2}(0)\right) \log (K L)-Z_{K L, j}+x_{i}\right)}\right) .
\end{aligned}
$$

Plugging (4.78) into (4.75) gives (4.74), with

$$
D_{K L}\left(A_{i}\right)=\sum_{j \in T_{i}^{(K L)}} e^{-2\left(\left(1+\sigma^{2}(0)\right) \log (K L)-Z_{K L, j}\right)} .
$$

Inserting (4.78) into (4.74), we obtain

$$
\lim _{\left(L, K, L^{\prime}, K^{\prime}\right) \Rightarrow \infty} \frac{\bar{\mu}_{L, K, L^{\prime}, K^{\prime}, \underline{A}}\left(\left(-\infty, x_{1}\right], \ldots,\left(-\infty, x_{p}\right]\right)}{\mathbb{E}\left[\exp \left(-\beta \sum_{i=1}^{p} D_{K L}\left(A_{i}\right) e^{-2 x_{i}}\right)\right]}=1 .
$$

Theorem 4.7 in combination with (4.80), implies that $\left\{D_{K L}\left(A_{i}\right): 1 \leq i \leq p\right\}$ converge weakly to random variables $\left\{D\left(A_{i}\right): 1 \leq i \leq p\right\}$, as $K L \rightarrow \infty$. Moreover, as the sequence of laws, $\left\{\bar{\mu}_{L, K, L^{\prime}, K^{\prime}, A}\right\}_{L, K, L^{\prime}, K^{\prime} \geq 0}$, is tight, it follows that almost surely, $D\left(A_{i}\right)>0$, for $i \in\{1, \ldots, p\}$. This concludes the proof.

Proof of Theorem 1.5: The random measure, $Y(\mathrm{~d} x)$, is completely characterized by its finitedimensional distributions over disjoint, open and non-empty subsets, $A_{1}, \ldots, A_{p} \subset[0,1]^{2}$, for $p \in \mathbb{N}$. Recall the defintion of the random vector, $\left(D_{K L}\left(A_{1}\right), \ldots D_{K L}\left(A_{p}\right)\right)$ in $(4.79)$ and the definition of the random measures, $\varphi_{N}^{\alpha}(\mathrm{d} x)$, in (1.10). On the one hand, we proved in the proof of Theorem 2.5 that the random vector, $\left(D_{K L}\left(A_{1}\right), \ldots D_{K L}\left(A_{p}\right)\right)$, converges, as $K L \rightarrow \infty$, to the random vector, $\left(D\left(A_{1}\right), \ldots, D\left(A_{p}\right)\right)$. By Theorem 2.5, this equals $\left(Y\left(A_{1}\right), \ldots, Y\left(A_{p}\right)\right)$, which also fixes the law of $Y$.

On the other hand, we observe that $\left(D_{K L}\left(A_{1}\right), \ldots D_{K L}\left(A_{p}\right)\right)$ equals $\left(\varphi_{K L}^{2 \sigma(0)}\left(A_{1}\right), \ldots, \varphi_{K L}^{2 \sigma(0)}\left(A_{p}\right)\right)$, which is known to converge to the Gaussian multiplicative measure associated with the continuum Gaussian free field on $[0,1]^{2}$ evaluated on $A_{1}, \ldots, A_{p},\left(\varphi_{\infty}^{2 \sigma(0)}\left(A_{1}\right), \ldots, \varphi_{\infty}^{2 \sigma(0)}\left(A_{p}\right)\right)$ (see (1.11) or cp. Kahane, 1985 and Shamov, 2016).

\section{Appendix}

5.1. Gaussian comparison. We need a vector version of Kahane's theorem.

Theorem 5.1. Let $f \in C^{2}\left(\mathbb{R}^{n} ; \mathbb{R}^{k}\right)$ with sub-Gaussian growth in every component of the second derivatives. Further let $\left\{X_{i}\right\}_{1 \leq i \leq n},\left\{Y_{i}\right\}_{1 \leq i \leq n}$ be two centred Gaussian fields satisfying

$$
\mathbb{E}\left[Y_{i} Y_{j}\right]>\mathbb{E}\left[X_{i} X_{j}\right] \Longrightarrow \frac{\partial f}{\partial x_{i} \partial x_{j}}(x) \geq 0, \quad x \in \mathbb{R},
$$

where the inequality is to be understood component-wise. Then,

$$
\mathbb{E}[f(Y)] \leq \mathbb{E}[f(X)],
$$

again to be understood as an inequality valid in each component.

Proof: The proof is an immediate adaptation of the original proof, as each component of $f$ is a function $f_{i} \in C^{2}\left(\mathbb{R}^{n}\right)$ with sub-Gaussian growth in its second derivatives, for which Kahane's theorem holds. In particular, each component of the map $f$ can be treated separately.

This allows us to deduce a vector version of Slepian's inequality. 
Theorem 5.2. Let $T$ be a countable index set, $\left\{X_{i}\right\}_{i \in T},\left\{Y_{i}\right\}_{i \in T}$ be two centred Gaussian fields satisfying

$$
\operatorname{Var}\left[X_{i}\right]=\operatorname{Var}\left[Y_{i}\right] \forall i \in T \quad \text { and } \quad \mathbb{E}\left[X_{i} X_{j}\right] \leq \mathbb{E}\left[Y_{i} Y_{j}\right], \forall i, j \in T .
$$

Then, for any disjoint collection of subsets $T_{1}, \ldots, T_{k} \subset T$ and real numbers $x_{1}, \ldots, x_{k} \in \mathbb{R}$,

$$
\mathbb{P}\left(\max _{i \in T_{1}} Y_{i} \leq x_{1}, \ldots, \max _{i \in T_{k}} Y_{i} \leq x_{k}\right) \leq \mathbb{P}\left(\max _{i \in T_{1}} X_{i} \leq x_{1}, \ldots, \max _{i \in T_{m}} X_{i} \leq x_{k}\right) .
$$

Proof: The proof is basically only a vector version of the original, which is why we just give a sketch. Assume for simplicity $|T|=n$. One takes a sequence of maps $f_{l}: \mathbb{R}^{n} \rightarrow \mathbb{R}^{k}$ of the form

$$
f_{l}=\left(\begin{array}{c}
\prod_{i \in A_{1}} g_{i}^{l}\left(x_{i}\right) \\
\prod_{i \in A_{2}} g_{i}^{l}\left(x_{i}\right) \\
\vdots \\
\prod_{i \in A_{k}} g_{i}^{l}\left(x_{i}\right)
\end{array}\right)
$$

where $g_{i}^{l}\left(x_{j}\right)$ are smooth, non-increasing and converge from above to $\mathbb{1}_{\left(-\infty, x_{j}\right]}$. One notices that the requirements of Theorem 5.1 are met, and an application of it finishes the proof.

\section{Acknowledgements}

The authors would like to thank an anonymous referee for a careful review of the preliminary version. We also gratefully acknowledge insightful discussions with Anton Bovier.

\section{References}

Aïdékon, E., Berestycki, J., Brunet, E., and Shi, Z. Branching Brownian motion seen from its tip. Probab. Theory Related Fields, 157 (1-2), 405-451 (2013). MR3101852.

Arguin, L.-P., Belius, D., and Bourgade, P. Maximum of the characteristic polynomial of random unitary matrices. Comm. Math. Phys., 349 (2), 703-751 (2017). MR3594368.

Arguin, L.-P., Belius, D., Bourgade, P., Radziwił 1, M., and Soundararajan, K. Maximum of the Riemann zeta function on a short interval of the critical line. Comm. Pure Appl. Math., 72 (3), 500-535 (2019). MR3911893.

Arguin, L.-P., Bovier, A., and Kistler, N. Genealogy of extremal particles of branching Brownian motion. Comm. Pure Appl. Math., 64 (12), 1647-1676 (2011). MR2838339.

Arguin, L.-P., Bovier, A., and Kistler, N. Poissonian statistics in the extremal process of branching Brownian motion. Ann. Appl. Probab., 22 (4), 1693-1711 (2012). MR2985174.

Arguin, L.-P., Bovier, A., and Kistler, N. The extremal process of branching Brownian motion. Probab. Theory Related Fields, 157 (3-4), 535-574 (2013). MR3129797.

Arguin, L.-P. and Ouimet, F. Extremes of the two-dimensional Gaussian free field with scaledependent variance. ALEA Lat. Am. J. Probab. Math. Stat., 13 (2), 779-808 (2016). MR3541850.

Arguin, L.-P. and Zindy, O. Poisson-Dirichlet statistics for the extremes of the two-dimensional discrete Gaussian free field. Electron. J. Probab., 20, no. 59, 19 (2015). MR3354619.

Biskup, M. and Louidor, O. Extreme local extrema of two-dimensional discrete Gaussian free field. Comm. Math. Phys., 345 (1), 271-304 (2016). MR3509015.

Biskup, M. and Louidor, O. Full extremal process, cluster law and freezing for the two-dimensional discrete Gaussian free field. Adv. Math., 330, 589-687 (2018). MR3787554.

Biskup, M. and Louidor, O. On intermediate level sets of two-dimensional discrete Gaussian free field. Ann. Inst. Henri Poincaré Probab. Stat., 55 (4), 1948-1987 (2019). MR4029145.

Bolthausen, E., Deuschel, J.-D., and Giacomin, G. Entropic repulsion and the maximum of the two-dimensional harmonic crystal. Ann. Probab., 29 (4), 1670-1692 (2001). MR1880237. 
Bolthausen, E., Deuschel, J. D., and Zeitouni, O. Recursions and tightness for the maximum of the discrete, two dimensional Gaussian free field. Electron. Commun. Probab., 16, 114-119 (2011). MR2772390.

Bovier, A. Gaussian processes on trees. From spin glasses to branching Brownian motion, volume 163 of Cambridge Studies in Advanced Mathematics. Cambridge University Press, Cambridge (2017). ISBN 978-1-107-16049-1. MR3618123.

Bovier, A. and Hartung, L. The extremal process of two-speed branching Brownian motion. Electron. J. Probab., 19, no. 18, 28 (2014). MR3164771.

Bovier, A. and Hartung, L. Variable speed branching Brownian motion 1. Extremal processes in the weak correlation regime. ALEA Lat. Am. J. Probab. Math. Stat., 12 (1), 261-291 (2015). MR3351476.

Bovier, A. and Hartung, L. Extended convergence of the extremal process of branching Brownian motion. Ann. Appl. Probab., 27 (3), 1756-1777 (2017). MR3678484.

Bovier, A. and Hartung, L. From 1 to 6: a finer analysis of perturbed branching Brownian motion. Comm. Pure Appl. Math., 73 (7), 1490-1525 (2020). MR4156608.

Bovier, A. and Kurkova, I. Derrida's generalized random energy models. II. Models with continuous hierarchies. Ann. Inst. H. Poincaré Probab. Statist., 40 (4), 481-495 (2004). MR2070335.

Bramson, M., Ding, J., and Zeitouni, O. Convergence in law of the maximum of the two-dimensional discrete Gaussian free field. Comm. Pure Appl. Math., 69 (1), 62-123 (2016). MR3433630.

Bramson, M. and Zeitouni, O. Tightness of the recentered maximum of the two-dimensional discrete Gaussian free field. Comm. Pure Appl. Math., 65 (1), 1-20 (2012). MR2846636.

Bramson, M. D. Maximal displacement of branching Brownian motion. Comm. Pure Appl. Math., 31 (5), 531-581 (1978). MR494541.

Cortines, A., Hartung, L., and Louidor, O. The structure of extreme level sets in branching Brownian motion. Ann. Probab., 47 (4), 2257-2302 (2019). MR3980921.

Daviaud, O. Extremes of the discrete two-dimensional Gaussian free field. Ann. Probab., 34 (3), 962-986 (2006). MR2243875.

Derrida, B. and Spohn, H. Polymers on disordered trees, spin glasses, and traveling waves. volume 51, pp. 817-840 (1988). New directions in statistical mechanics (Santa Barbara, CA, 1987). MR971033.

Ding, J. Exponential and double exponential tails for maximum of two-dimensional discrete Gaussian free field. Probab. Theory Related Fields, 157 (1-2), 285-299 (2013). MR3101848.

Ding, J., Roy, R., and Zeitouni, O. Convergence of the centered maximum of log-correlated Gaussian fields. Ann. Probab., 45 (6A), 3886-3928 (2017). MR3729618.

Ding, J. and Zeitouni, O. Extreme values for two-dimensional discrete Gaussian free field. Ann. Probab., 42 (4), 1480-1515 (2014). MR3262484.

Fang, M. and Zeitouni, O. Slowdown for time inhomogeneous branching Brownian motion. J. Stat. Phys., 149 (1), 1-9 (2012). MR2981635.

Fels, M. Extremes of the 2d scale-inhomogeneous discrete Gaussian free field: Sub-leading order and exponential tails. ArXiv Mathematics e-prints (2019). arXiv: 1910.09915v4.

Fels, M. and Hartung, L. Extremes of the 2d scale-inhomogeneous discrete Gaussian free field: Convergence of the maximum in the regime of weak correlations. ArXiv Mathematics e-prints (2019). arXiv: 1912.13184v3.

Fyodorov, Y. V. Complexity of random energy landscapes, glass transition, and absolute value of the spectral determinant of random matrices. Phys. Rev. Lett., 92 (24), 240601, 4 (2004). MR2115095.

Grimmett, G. Percolation, volume 321 of Grundlehren der Mathematischen Wissenschaften [Fundamental Principles of Mathematical Sciences]. Springer-Verlag, Berlin, second edition (1999). ISBN 3-540-64902-6. MR1707339.

Kahane, J.-P. Sur le chaos multiplicatif. Ann. Sci. Math. Québec, 9 (2), 105-150 (1985). MR829798. 
Kistler, N., Schertzer, A., and Schmidt, M. A. Oriented first passage percolation in the mean field limit, 2. The extremal process. Ann. Appl. Probab., 30 (2), 788-811 (2020). MR4108122.

Lalley, S. P. and Sellke, T. A conditional limit theorem for the frontier of a branching Brownian motion. Ann. Probab., 15 (3), 1052-1061 (1987). MR893913.

Lawler, G. F. and Limic, V. Random walk: a modern introduction, volume 123 of Cambridge Studies in Advanced Mathematics. Cambridge University Press, Cambridge (2010). ISBN 978-0521-51918-2. MR2677157.

Maillard, P. and Zeitouni, O. Slowdown in branching Brownian motion with inhomogeneous variance. Ann. Inst. Henri Poincaré Probab. Stat., 52 (3), 1144-1160 (2016). MR3531703.

Mallein, B. Maximal displacement in a branching random walk through interfaces. Electron. $J$. Probab., 20, no. 68, 40 (2015). MR3361256.

Mallein, B. Genealogy of the extremal process of the branching random walk. ALEA Lat. Am. J. Probab. Math. Stat., 15 (2), 1065-1087 (2018). MR3852245.

Rhodes, R. and Vargas, V. Gaussian multiplicative chaos and applications: a review. Probab. Surv., 11, 315-392 (2014). MR3274356.

Shamov, A. On Gaussian multiplicative chaos. J. Funct. Anal., 270 (9), 3224-3261 (2016). MR3475456. 